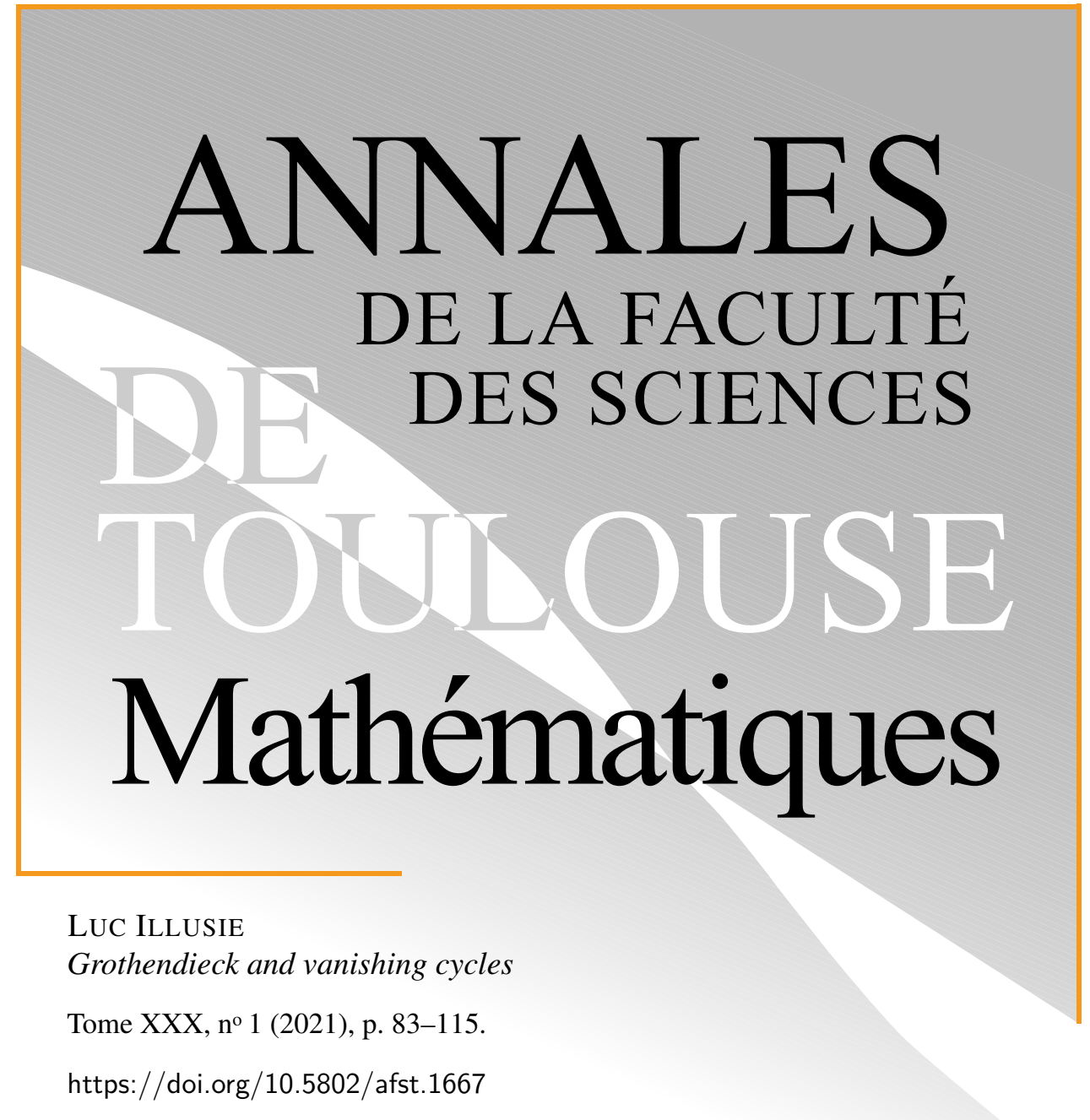

(c) Université Paul Sabatier, Toulouse, 2021.

L'accès aux articles de la revue «Annales de la faculté des sciences de Toulouse Mathématiques » (http://afst.centre-mersenne.org/) implique l'accord avec les conditions générales d'utilisation (http://afst.centre-mersenne.org/legal/). Les articles sont publiés sous la license CC-BY 4.0.

(c) $\underset{B Y}{(1)}$

Publication membre du centre

Mersenne pour l'édition scientifique ouverte MERSENNE http://www.centre-mersenne.org/ 


\title{
Grothendieck and vanishing cycles ${ }^{(*)}$
}

\author{
LUC ILLUSIE ${ }^{(1)}$
}

To the memory of Michel Raynaud

\begin{abstract}
This is a survey of classical results of Grothendieck on vanishing cycles, such as the local monodromy theorem and his monodromy pairing for abelian varieties over local fields $([22, \mathrm{IX}])$. We discuss related current developments and questions. At the end, we include the proof of an unpublished result of Gabber giving a refined bound for the exponent of unipotence of the local monodromy for torsion coefficients.

RÉsumé. - Le présent texte est un exposé de résultats classiques de Grothendieck sur les cycles évanescents, tels que le théorème de monodromie locale et l'accouplement de monodromie pour les variétés abéliennes sur les corps locaux ([22, IX]). Nous présentons quelques développements récents et questions qui y sont liés. La dernière section est consacrée à la démonstration d'un résultat inédit de Gabber donnant une borne raffinée pour l'exposant d'unipotence de la monodromie locale pour des coefficients de torsion.
\end{abstract}

Grothendieck's first mention of vanishing cycles is in a letter to Serre, dated Oct. 30, 1964 ([15, p. 214]). He considers a regular, proper, and flat curve $X$ over a strictly local trait $S=(S, s, \eta)$, whose generic fiber is smooth and whose reduced special fiber is a divisor with normal crossings. He analyses the difference between the (étale) cohomology of the special fiber $H^{*}\left(X_{s}\right)$ and that of the generic geometric fiber $H^{*}\left(X_{\bar{\eta}}\right)$, the coefficients ring being $\mathbf{Z}_{\ell}, \ell$ a prime number invertible on $S$. A little more precisely, assuming that

${ }^{(*)}$ Reçu le 12 octobre 2018, accepté le 27 mai 2019.

Keywords: Étale cohomology, monodromy, Milnor fiber, nearby and vanishing cycles, alteration, hypercovering, semistable reduction, intersection complex, abelian scheme, Picard functor, Jacobian, Néron model, Picard-Lefschetz formula, $\ell$-adic sheaf.

2020 Mathematics Subject Classification: 01A65, 11F80, 11G10, 13D09, 14-03, 14D05, 14F20, 14G20, 14K30, 14H25, 14L05, 14L15, 32L55.

(1) Laboratoire de Mathématiques d'Orsay, CNRS, Université Paris-Saclay, 91405

Orsay Cedex, France — luc.illusie@wanadoo.fr

Article proposé par Damian Rössler. 
the action of the inertia group $I$ on $H^{*}\left(X_{\bar{\eta}}\right)$ is tame, he shows that the defect of the specialization map $H^{*}\left(X_{s}\right) \rightarrow H^{*}\left(X_{\bar{\eta}}\right)^{(2)}$ to be an isomorphism is controlled by certain groups (the vanishing cycles groups), that he estimates. He deduces that there exists an open subgroup $I_{1}$ of $I$ such that, for all $g \in I_{1},(g-1)^{2}$ acts trivially on $H^{*}\left(X_{\bar{\eta}}\right)$, a key step in his proof of the semistable reduction theorem for abelian varieties.

We will recall this proof in Section 3, after a quick review, in Sections 1 and 2, of the definition and basic properties of nearby and vanishing cycles, and Grothendieck's geometric local monodromy theorem. In Section 4 we discuss Grothendieck's monodromy pairing for abelian varieties over local fields, a complement to his semistable reduction theorem. In Section 5 we say a few words about the developments that arose from Grothendieck's work and questions. In Section 6 we give a quick update on some of the topics of Sections 1-4. The last section is devoted to the proof of Theorem 2.3, a result due to Gabber.

Additional references. Here are a few references that could help the reader who is not familiar with the topics discussed in this report. The formalism of nearby and vanishing cycles is presented in Deligne's exposés I, XIII, XIV and XV of [22]. Fundamental theorems such as constructibility and compatibility with duality are proved in [20, Théorèmes de finitude en cohomologie $\ell$-adique], [7], [34]. The reader could also consult Lei Fu's monograph [25]. Further references are provided in 6.2. For basics on abelian schemes, including the Picard functor and duality, see [24]. Néron models are treated in Bosch-Lütkebohmert-Raynaud's book [14].

Acknowledgments. This is a slightly expanded version of notes of a talk given on June 17, 2015, at the conference Grothendieck2015 at the University of Montpellier, and on November 13, 2015, at the conference Moduli Spaces and Arithmetic Geometry at the Lorentz Center in Leiden. I wish to thank these institutions for their invitation

I thank Brian Conrad, Ofer Gabber, Cédric Pépin and Takeshi Saito for helpful remarks and discussions on a first draft of these notes. I am especially indebted to Ofer Gabber for detecting an error in my account of his proof of Theorem 2.3 in a later version of this text, and helping me correct it. I thank Weizhe Zheng for constructive criticism on one of the last versions. Finally, I am very grateful to the referee for carefully reading the manuscript, correcting a few inaccuracies, and making useful comments and suggestions.

(2) This is the composition of the inverse $H^{*}\left(X_{s}\right) \stackrel{\sim}{\rightarrow} H^{*}(X)$ of the proper base change isomorphism and the restriction map $H^{*}(X) \rightarrow H^{*}\left(X_{\bar{\eta}}\right)$. 


\section{Nearby and vanishing cycles}

1.1. In [22] Grothendieck introduced and studied the functors $R \Psi$ and $R \Phi$, both in the context of Betti cohomology and that of étale cohomology. He called them functors of vanishing cycles, but in the 1970's it became customary to call the former one the functor of nearby cycles, the name vanishing cycles being reserved to the latter one. Let me recall their definition in the étale setup, as described by Deligne in ([22, XIII $])$.

Let $S=(S, s, \eta)$ be a henselian trait, with closed point $s$, and generic point $\eta$. Let $\bar{s}$ be a geometric point of $S$ over $s$, and $\bar{\eta}$ a separable closure of the generic point $\widetilde{\eta}$ of the strict henselization $\widetilde{S}=S_{(\bar{s})}$ of $S$ at $\bar{s}$. We have a commutative diagram with cartesian squares

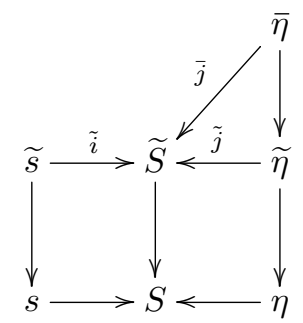

where $\widetilde{s}$, the closed point of $\widetilde{S}$, is a separable closure of $s$ in $\bar{s}$. For a morphism $f: X \rightarrow S$, we get morphisms deduced by base change

$$
X_{\tilde{s}} \stackrel{\tilde{i}}{\longrightarrow} X_{\tilde{S}} \stackrel{\bar{j}}{\leftrightarrows} X_{\bar{\eta}} \text {. }
$$

Let us choose a ring of coefficients $\Lambda=\mathbf{Z} / \ell^{\nu} \mathbf{Z}, \nu \geqslant 1$, with $\ell$ a prime number invertible on $S$. Other choices are possible, e.g., $\mathbf{Z}_{\ell}, \mathbf{Q}_{\ell}$, or $\overline{\mathbf{Q}}_{\ell}$ (when one works with schemes of finite type over $S^{(3)}$ ). We will write $D(\cdot)$ for $D(\cdot, \Lambda)$. For $K \in D^{+}\left(X_{\eta}\right)$, the nearby cycles complex of $K$ is

$$
R \Psi_{f}(K):=\widetilde{i}^{*} R \bar{j}_{*}\left(K \mid X_{\bar{\eta}}\right) .
$$

This is an object of $D^{+}\left(X_{\tilde{s}}\right)$, more precisely an object of the derived category of sheaves of $\Lambda$-modules on $X_{\tilde{s}}$ equipped with a continuous action of the Galois group $\operatorname{Gal}(\bar{\eta} / \eta)$, compatible with that on $X_{\tilde{s}}$. For $K \in D^{+}(X)$, the complex of vanishing cycles $R \Phi_{f}(K)$ is the cone of the natural morphism $\widetilde{i}^{*} K \rightarrow R \Psi_{f}\left(K \mid X_{\eta}\right)$, i.e., we have a distinguished triangle in the category just mentioned,

$$
\widetilde{i}^{*} K \longrightarrow R \Psi_{f}\left(K \mid X_{\eta}\right) \longrightarrow R \Phi_{f}(K) \longrightarrow .
$$

(3) This is to ensure that $D_{c}^{b}$ is stable under the six operations. 
If $\bar{x}$ is a geometric point of $X$ over $\bar{s}$, the stalk of $R \Psi_{f}(K)$ at $\bar{x}$ is

$$
R \Psi_{f}(K)_{\bar{x}}=R \Gamma\left(\left(X_{(\bar{x})}\right)_{\bar{\eta}}, K\right) .
$$

The scheme $\left(X_{(\bar{x})}\right)_{\bar{\eta}}$, geometric generic fiber of the strict localization of $X$ at $\bar{x}$, plays the role of a Milnor fiber of $f$ at $\bar{x}$. We sometimes write $R \Psi_{X}$ (resp. $R \Phi_{X}$ ) for $R \Psi_{f}$ (resp. $R \Phi_{f}$ ), and drop the subscript $X$ when no confusion can arise.

1.2. The main functoriality properties of these functors are the following. Consider a commutative triangle

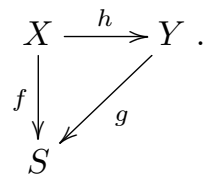

(a) If $h$ is smooth, the natural map

$$
h^{*} R \Psi_{Y} \longrightarrow R \Psi_{X} h^{*}
$$

is an isomorphism. In particular (taking $Y=S$ ), if $f$ is smooth, then $R \Phi_{X}(\Lambda)=0$. So, in general, $R \Phi_{X}(\Lambda)$ is concentrated on the non-smoothness locus of $X / S$.

(b) If $h$ is proper, the natural map ${ }^{(4)}$

$$
R h_{*} R \Psi_{X} \longrightarrow R \Psi_{Y} R h_{*}
$$

is an isomorphism. In particular (taking $Y=S$ ), if $f$ is proper, for $K \in D^{+}\left(X_{\eta}\right)$, we have a canonical isomorphism (compatible with the Galois actions)

$$
R \Gamma\left(X_{\tilde{s}}, R \Psi_{X} K\right) \stackrel{\sim}{\longrightarrow} R \Gamma\left(X_{\bar{\eta}}, K\right) .
$$

The triangle (1.3) thus gives rise to a long exact sequence

$$
\begin{aligned}
\cdots \longrightarrow H^{i-1}\left(X_{\tilde{s}}, R \Phi_{X}(K)\right) \longrightarrow H^{i}\left(X_{\tilde{s}}, K\right) \stackrel{\mathrm{sp}}{\longrightarrow} H^{i}\left(X_{\bar{\eta}}, K\right) \\
\longrightarrow H^{i}\left(X_{\tilde{s}}, R \Phi_{X}(K)\right) \longrightarrow \cdots,
\end{aligned}
$$

where sp is the specialization map, generalizing that considered in the introduction.

It was later proved by Deligne $([20$, Th. finitude $])$ that, for $X$ of finite type over $S$ and $K \in D_{c}^{b}\left(X_{\eta}\right), R \Psi_{f} K$ is in $D_{c}^{b}\left(X_{\tilde{s}}\right)$ (where $D_{c}^{b}(\cdot)$ means the full subcategory of $D(\cdot)$ consisting of complexes with bounded and constructible cohomology).

(4) In (a) and (b), there are obvious abuses of notation for $h^{*}$ and $h_{*}$. 


\section{The geometric local monodromy theorem}

In [22] Grothendieck proved the following theorem:

THEOREM 2.1. - Let $S=(S, s, \eta)$ be as in 1.1, and $X_{\eta}$ be separated and of finite type over $\eta$. Let $I=\operatorname{Gal}(\bar{\eta} / \widetilde{\eta}) \subset \operatorname{Gal}(\bar{\eta} / \eta)$ be the inertia group. Then there exists an open subgroup $I_{1} \subset I$ such that, for all $g \in I_{1}$ and all $i \in \mathbf{Z}, g$ acts unipotently on $H_{c}^{i}\left(X_{\bar{\eta}}, \mathbf{Q}_{\ell}\right)$.

The main ingredient in his proof was his arithmetic local monodromy theorem ([68, Appendix]):

THEOREM 2.2. - Assume that no finite extension of $k(s)$ contains all roots of unity of order a power of $\ell$. Let $\rho: \operatorname{Gal}(\bar{\eta} / \eta) \rightarrow \operatorname{GL}(V)$ be a continuous representation into a finite dimensional $\mathbf{Q}_{\ell}$-vector space $V$. Then there exists an open subgroup $I_{1}$ of $I$ such that, for all $g \in I_{1}, \rho(g)$ is unipotent.

The proof of Theorem 2.2 is an elegant, elementary exercise. Once we have reduced to the case where the image of $\rho$ is contained in $1+\ell^{2} M_{n}\left(\mathbf{Z}_{\ell}\right)$, the whole inertia group $I$ acts unipotently. Indeed, $I$ acts through its $\ell$-primary tame quotient $t_{\ell}: I \rightarrow \mathbf{Z}_{\ell}(1)$, and Grothendieck exploits the strong action of the arithmetic Galois group $G_{k}=\operatorname{Gal}(\widetilde{s} / s)$ on $\mathbf{Z}_{\ell}(1)$ by conjugation, given by $g \sigma g^{-1}=\sigma^{\chi(g)}$, where $\chi: G_{k} \rightarrow \mathbf{Z}_{\ell}^{*}$ is the cyclotomic character $\left(\ell^{2}\right.$ ensures that exponential and logarithm are defined and inverse to each other).

The deduction of Theorem 2.1 from Theorem 2.2 is more difficult. It uses Néron's desingularization, and a spreading out argument to reduce to the case where the residue field is radicial over a finite type extension of $\mathbf{F}_{p}$, see $([22, \mathrm{I} 1.3])$. Once the finite generation of the groups $H^{i}\left(X_{\bar{\eta}}, \Lambda\right)$ (for $\Lambda=\mathbf{Z} / \ell^{\nu} \mathbf{Z}$ ) (and generic constructibility of direct images) was known ([20, Th. finitude]), the same reduction worked - hence the conclusion of Theorem 2.1 held - for $H^{i}\left(X_{\bar{\eta}}, \mathbf{Q}_{\ell}\right)$ as well.

Grothendieck gave a conditional, alternate proof of Theorem 2.1, based on the formalism of Section 1. It assumed the validity (in certain degrees and dimensions) of resolution of singularities and of his absolute purity conjecture $([31, \mathrm{I}])^{(5)}$. This was the case for $s$ of characteristic zero, or $\operatorname{dim}\left(X_{\eta}\right) \leqslant 1$, or $i \leqslant 1$. The advantage of the method is that it gave bounds on the exponent of unipotence $n(g)$ of $g \in I_{1}$ acting on $H^{i}$, i.e., the smallest integer $n \geqslant 0$ such that $(g-1)^{n+1}=0$. For example, for $i=1$, one gets $n(g) \leqslant 1$. Resolution is still an open problem, but the absolute purity conjecture was proved by

(5) One form of this conjecture is that if $j: U=X-D \hookrightarrow X$ is the inclusion of the complement of a regular divisor $D$ in a regular scheme $X$, and if $\ell$ is invertible on $X$, then $R^{q} j_{*} \Lambda$ is $\Lambda$ for $q=0, \Lambda_{D}(-1)$ for $q=1$, and 0 for $q>1$. 
Rapoport-Zink in the situation arising from a semistable reduction $[56]^{(6)}$, and thanks to de Jong's alteration theorems [45], it was possible to make Grothendieck's argument work in general. As Deligne observed, a by-product of this argument was that the open subgroup $I_{1}$ in Theorem 2.1 (and its variant for $H^{i}$ ) can be chosen independent of $\ell$ (see Berthelot's Bourbaki exposé [10]). With more work, one can also get a general bound for $n(g)$, valid also for torsion coefficients $\Lambda=\mathbf{Z} / \ell^{\nu} \mathbf{Z}$, namely, one has the following result:

THEOREM 2.3. - Let $\Lambda=\mathbf{Z} / \ell^{\nu} \mathbf{Z}$. With the assumptions and notation of Theorem 2.1 for $S$ and $X_{\eta}$, there exists an open subgroup $I_{1} \subset I$, independent of $\ell$, such that, for all $g \in I_{1}$ and all $i \in \mathbf{N},(g-1)^{i+1}=0$ on $H_{c}^{i}\left(X_{\bar{\eta}}, \Lambda\right)$ (resp. $\left.H^{i}\left(X_{\bar{\eta}}, \Lambda\right)\right)$.

This result is due to Gabber. See Section 7 for the proof.

Remark 2.4. - For smooth, projective, geometrically connected $X_{\eta} / \eta$, explicit uniform bounds for the index of $I_{1}$ in $I$ for the action of $I$ on $H^{i}\left(X_{\bar{\eta}}, \mathbf{Q}_{\ell}\right)$ in terms of the Betti numbers of $X_{\eta}$ and numerical invariants associated with a very ample line bundle on $X_{\eta}$ were obtained by Umezaki [75].

Remark 2.5. - Suppose $X_{\eta} / \eta$ is proper and equidimensional of dimension $d$. Let $I H^{i}\left(X_{\bar{\eta}}, \Lambda\right):=H^{i}\left(X_{\bar{\eta}}, \underline{I C}[-d]\right)$, where $\underline{I C}$ is the pull-back to $X_{\bar{\eta}}$ of the intersection complex $\underline{I C}_{X_{\eta}}:=j_{!_{*}}\left(\Lambda_{U}[d]\right.$ ) (where $j: U \hookrightarrow X_{\eta}$ is the inclusion of a dense open subscheme such that $\left(U_{\bar{\eta}}\right)_{\text {red }}$ is smooth). One can ask whether there exists an open subgroup $I_{1}$ of $I$, independent of $\ell$, such that, for all $g \in I_{1}$ and all $i \in \mathbf{N},(g-1)^{i+1}=0$ on $I H^{i}\left(X_{\bar{\eta}}, \Lambda\right)$. The answer is yes for $\Lambda=\mathbf{Q}_{\ell}$ or $\overline{\mathbf{Q}}_{\ell}$. Indeed, by de Jong [45], after replacing $\eta$ by a finite extension, one can find an alteration $h: Z \rightarrow X_{\eta}$, with $Z$ proper and smooth over $\eta$ (and purely of dimension $d$ ). By the generalization of the decomposition theorem of Beilinson-Bernstein-Deligne-Gabber ([7, 5.3, 5.4]) given in $[70,5.1], \underline{I C}$ is a direct summand of $R h_{*} \Lambda_{Z_{\bar{\eta}}}[d]$. I don't know the answer for $\Lambda=\mathbf{Z} / \ell^{\nu} \mathbf{Z}$.

2.1. The main step in Grothendieck's geometric proof is a calculation of the stalks of the tame nearby cycles groups $R^{q} \Psi_{X}(\Lambda)_{\mathrm{t}}$ (for $\Lambda=\mathbf{Z} / \ell^{\nu} \mathbf{Z}$ ), in a situation of quasi-semistable reduction (assuming that absolute purity is available - which is the case today). Let me recall the definition of these groups. In (1.1), $k(\widetilde{\eta})$ is the maximal unramified extension of $k(\eta)$ contained in $k(\bar{\eta})$. Let $k\left(\eta_{\mathrm{t}}\right)$ be the maximal tame extension of $k(\eta)$ contained in $k(\bar{\eta})$, i.e., $k\left(\eta_{\mathrm{t}}\right)=\lim _{\longrightarrow} k(\widetilde{\eta})\left[\pi^{1 / n}\right]$, where $\pi$ is a uniformizing parameter of $\widetilde{S}$, and $n$

(6) It was later proved by Gabber in general [26], but the semistable reduction case sufficed. 
runs through the integers $\geqslant 1$ prime to the characteristic exponent $p$ of $k(s)$. Then $P:=\operatorname{Gal}\left(\bar{\eta} / \eta_{\mathrm{t}}\right)$ is the wild inertia subgroup of $I=\operatorname{Gal}(\bar{\eta} / \widetilde{\eta})$, and

$$
I_{\mathrm{t}}=\operatorname{Gal}\left(\eta_{\mathrm{t}} / \widetilde{\eta}\right)=\widehat{\mathbf{Z}}^{\prime}(1):=\lim _{(n, p)=1} \mathbf{Z} / n(1)
$$

its tame quotient. Replacing the upper part of (1.1) by

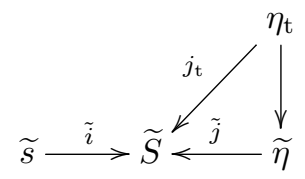

one defines, for $X$ over $\eta$, and $K \in D^{+}(X)$, the tame nearby cycles complex

$$
R \Psi_{f}(K)_{\mathrm{t}}:=\widetilde{i}^{*} R j_{\mathrm{t} *}\left(K \mid X_{\eta_{\mathrm{t}}}\right)
$$

As $P$ is a pro-p-group, the functor $(\cdot)^{P}$ (invariants under $P$ ) is exact, and one has

$$
R \Psi_{f}(K)_{\mathrm{t}} \stackrel{\sim}{\longrightarrow} R \Psi_{f}(K)^{P}
$$

For $X / S$ and $K \in D^{+}(X)$, one defines the tame vanishing cycles complex $R \Phi_{f}(K)_{\mathrm{t}}$ similarly to $R \Phi_{f}(K)$. One has a variant of (1.4):

$$
R \Psi_{f}(K)_{\mathrm{t}, \bar{x}}=R \Gamma\left(\left(X_{(\bar{x})}\right)_{\eta_{\mathrm{t}}}, K\right),
$$

with the Milnor fiber replaced by the tame one $\left(X_{(\bar{x})}\right)_{\eta_{\mathrm{t}}}$.

2.2. Assume now that $X$ is regular, flat and of finite type over $S$, the generic fiber $X_{\eta}$ is smooth, and the reduced special fiber $\left(X_{s}\right)_{\text {red }}$ is a divisor with normal crossings. Let $\bar{x}$ be a geometric point of $X$ over $\bar{s}$, let $\left(D_{i}\right)_{1 \leqslant i \leqslant r}$ be the

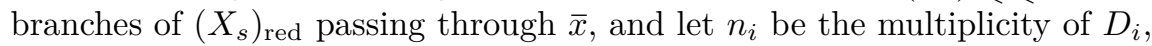
i.e., $X$ is locally defined near $\bar{x}$ by an equation of the form $u \prod_{1 \leqslant i \leqslant r} t_{i}^{n_{i}}=\pi$, where $\pi$ is a uniformizing parameter of $S$, the $t_{i}$ 's are part of a system of regular parameters at the strict localization of $X$ at $\bar{x}$, and $u$ is a unit at $\bar{x}$. Then ([22, I 3.3]) the stalks of the groups $\left(R^{q} \Psi \Lambda\right)_{\mathrm{t}}$ at $\bar{x}$ are given by

$$
\left(R^{q} \Psi \Lambda\right)_{\mathrm{t}, \bar{x}}=\Lambda[(\mathbf{Z} / d \mathbf{Z})(1)] \otimes_{\mathbf{Z}} \Lambda^{q}(C(-1)),
$$

where

$$
C=\operatorname{Ker}\left(\left(n_{1}, \ldots, n_{r}\right): \mathbf{Z}^{r} \longrightarrow \mathbf{Z}\right),
$$

and $\operatorname{gcd}\left(n_{i}\right)=d p^{m}$, with $(d, p)=1$. The inertia group $I$ acts on them via its permutation action on $(\mathbf{Z} / d \mathbf{Z})(1)$ through the composition $I \rightarrow$ $I_{\mathrm{t}} \rightarrow(\mathbf{Z} / d \mathbf{Z})(1)$. The reason for this is that, because of absolute purity, on the cohomological level the tame Milnor fiber $\left(X_{(\bar{x})}\right)_{\eta_{\mathrm{t}}}(2.4)$ behaves like the (prime to $p$ ) homotopy fiber of the homomorphism $\left(S^{1}\right)^{r} \rightarrow S^{1}$, $\left(x_{1}, \ldots, x_{r}\right) \mapsto \prod x_{i}^{n_{i}}$, i.e., the wreath product $\operatorname{Ker}\left(\left(S^{1}\right)^{r} \rightarrow S^{1}\right) \rtimes(\mathbf{Z} / d \mathbf{Z})(1)$. An immediate consequence is: 
COROllary 2.6. - Under the assumptions of Theorem 2.2, there exists an open subgroup $I_{1}$ of $I$ such that, for all $q \in \mathbf{Z}, I_{1}$ acts trivially on $\left(R^{q} \Psi \Lambda\right)_{\mathrm{t}}$.

At the time of SGA 7, the proof of (2.5) was conditional to the validity of the absolute purity conjecture, which was known only in certain cases (e.g., in equal characteristic zero, and for $q \leqslant 1$ in the notation of footnote (5)). Nevertheless, this, together with the cases where resolution of singularities was known, enabled Grothendieck to show that Theorem 2.3 holds if $X_{\eta}$ is proper and smooth and either $S$ is the localization of a smooth curve over $\mathbf{C}$, or $i \leqslant 1$. Using the formalism of 1.2 (or rather its analogue in the complex case), he also deduced from Corollary 2.6 a positive answer to Milnor's question $([51 \text {, footnote p. } 72])^{(7)}$ on the quasi-unipotence of the monodromy of isolated singularities (a question that had been one of the motivations for his theory of the functors $R \Psi$ and $R \Phi)$. In fact, because of the now known validity of the absolute purity conjecture, (2.5) holds unconditionally, and, moreover, in the case of semistable reduction, $R^{q} \Psi \Lambda=$ $\left(R^{q} \Psi \Lambda\right)_{\mathrm{t}}$, see 6.3 .

Here is a sketch of Grothendieck's answer to Milnor's question. As we have an isolated critical point, by a theorem of Arnol'd-Artin-Mather-Tougeron (see [1] for references), $\widetilde{H}^{n}\left(M_{f}\right)$ depends only on a sufficiently high order jet of $f$, so, instead of the original analytic situation, we can consider an algebraic one, namely a smooth curve $S$ over $\mathbf{C}$, with a closed point $s$, a smooth scheme $X / \mathbf{C}$, and a morphism $f: X \rightarrow S$, smooth outside a closed point $x$ of the special fiber $X_{s}$. By Hironaka, we can find a proper map $h: X^{\prime} \rightarrow X$, with $X^{\prime} / \mathbf{C}$ smooth, inducing an isomorphism outside $X_{s}$ and such that $\left(X_{s}^{\prime}\right)_{\text {red }}$ is a divisor with normal crossings. Let $f^{\prime}=f h$. By Corollary 2.6, the action of a generator $T$ of the local fundamental group of $S$ at $s$ on $R \Psi_{f^{\prime}} \mathbf{Z}$ is quasi-unipotent. By (the complex analytic analogue of) (1.6), $R \Psi_{f} \mathbf{Z}=R h_{*} R \Psi_{f} \mathbf{Z}$, so $T$ acts quasi-unipotently on $R \Psi_{f} \mathbf{Z}$, hence on $R \Phi_{f} \mathbf{Z}$, which is concentrated at $x$, i.e., equal to $i_{x *}\left(R \Phi_{f} \mathbf{Z}\right)_{x}$, where $i_{x}$ : $\{x\} \hookrightarrow X_{s}$.

\section{The semistable reduction theorem for abelian varieties}

As an abelian variety over an algebraically closed field is a quotient of a Jacobian, from $n(g) \leqslant 1$ for $i=1$ we get:

(7) i.e., for a holomorphic germ $f:\left(\mathbf{C}^{n+1}, 0\right) \rightarrow(\mathbf{C}, 0)$ having an isolated critical point at 0 , the eigenvalues of the monodromy $T$ on $\widetilde{H}^{n}\left(M_{f}\right)$ are roots of $1\left(M_{f}\right.$ the Milnor fiber at 0 , where $\widetilde{H}^{i}=\operatorname{Coker}\left(H^{i}(\mathrm{pt}) \rightarrow H^{i}\right)$. Here $\widetilde{H}^{n}\left(M_{f}\right)=R^{n} \Phi_{f}(\mathbf{Z})_{0}$. 
THEOREM 3.1. - With the notation of Theorem 2.1, let $A_{\eta}$ be an abelian variety over $\eta$. Then there exists an open subgroup $I_{1}$ of I such that, for all $g \in I_{1},(g-1)^{2}=0$ on $H^{1}\left(A_{\bar{\eta}}\right)$.

As mentioned in the introduction, this was the crucial tool enabling Grothendieck to prove the following theorem (semistable reduction theorem for abelian varieties) ([22, IX 3.6]):

THEOREM 3.2. - With the notation of Theorem 3.1, there exists a finite extension $\eta_{1}$ of $\eta$ such that $A_{\eta_{1}}$ has semistable reduction ${ }^{(8)}$ over the normalization $\left(S_{1}, s_{1}, \eta_{1}\right)$ of $S$ in $\eta_{1}$, i.e., if $A_{1} / S_{1}$ is the Néron model of $A_{\eta_{1}}$, the connected component $\left(A_{1}\right)_{s_{1}}^{0}$ of its special fiber is an extension of an abelian variety by a torus.

The proof of Theorem 3.2 occupies over 300 pages in ([22, VII, VIII, IX]). However, the idea is quite simple.

First of all, one rephrases Theorem 3.1 in terms of the Tate module of $A_{\eta}$,

$$
T_{\ell}\left(A_{\eta}\right)=\lim _{\longleftarrow} A_{\bar{\eta}}\left[\ell^{n}\right]
$$

where $\left[\ell^{n}\right]$ means the kernel of the multiplication by $\ell^{n}$, a free $\mathbf{Z}_{\ell}$-module of rank $2 g$, where $g$ is the dimension of $A_{\eta}$, equipped with a continuous action

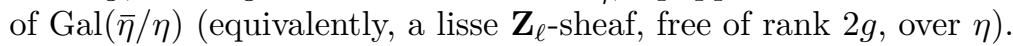

From now on, let us work with $\Lambda=\mathbf{Z}_{\ell}$.

By Serre-Lang,

$$
H^{1}\left(A_{\bar{\eta}}\right)=T_{\ell}\left(A_{\eta}\right)^{\vee}\left(:=\operatorname{Hom}\left(T_{\ell}\left(A_{\bar{\eta}}\right), \mathbf{Z}_{\ell}\right)\right)
$$

as Galois modules. Hence, in the notation of Theorem 3.1, for all $g \in I_{1}$, $(g-1)^{2}=0$ on $T_{\ell}\left(A_{\eta}\right)$. To prove Theorem 3.2 it therefore suffices to prove the following theorem (cohomological criterion for semistable reduction) ([22, IX, 3.5]):

THEOREM 3.3. - In the situation of Theorem 3.1, assume that for all $g \in I,(g-1)^{2}=0$ on $T_{\ell}\left(A_{\eta}\right)$. Then $A_{\eta}$ has semistable reduction over $S$.

3.1. The main ingredient in the proof of Theorem 3.3 is the so-called orhogonality theorem, which I will now recall. In the situation of Theorem 3.1, let $A$ be the Néron model of $A_{\eta}$ over $S$ (so that $A_{\eta}$ is the generic fiber of $A$ ) ([14, 1.2, 1.3]). The Tate module $T_{\ell}\left(A_{\eta}\right)$ admits a $\operatorname{Gal}(\bar{\eta} / \eta)$-equivariant 2step filtration

$$
T_{\ell}\left(A_{\eta}\right)^{\mathrm{t}} \subset T_{\ell}\left(A_{\eta}\right)^{\mathrm{f}} \subset T_{\ell}\left(A_{\eta}\right),
$$

(8) Today one often prefers to say "semi-abelian reduction", to avoid confusion with semistable reduction as a scheme. 
where, as a Galois module, $T_{\ell}\left(A_{\eta}\right)^{\mathrm{f}}=T_{\ell}\left(A_{\eta}\right)^{I}$ is the fixed part under $I$, which, by the universal property of the Néron model, is also canonically isomorphic to $T_{\ell}\left(A_{s}\right)=T_{\ell}\left(A_{s}^{0}\right)$, and $T_{\ell}\left(A_{\eta}\right)^{\mathrm{t}}$ the toric part, i.e., $T_{\ell}(T)$, where $T$ is the maximal subtorus of $A_{s}^{0}$. In ([22], IX), Grothendieck likes to write (3.1) in the form

$$
W \subset V \subset U \text {. }
$$

This is a filtration by free, finitely generated $\mathbf{Z}_{\ell^{-}}$modules, and the quotients $U / V, V / W$ are torsion-free ${ }^{(9)}$. Let $A_{\eta}^{\prime}=\mathcal{E}_{\mathrm{xt}^{1}}\left(A_{\eta}, \mathbf{G}_{m}\right)$ be the dual abelian variety (cf. [22, VIII 3.2] $)^{(10)}, A^{\prime}$ its Néron model, and let $\left(W^{\prime} \subset V^{\prime} \subset U^{\prime}\right)$ be the corresponding filtration of $U^{\prime}=T_{\ell}\left(A_{\eta}^{\prime}\right)$. The Poincaré bi-extension of $A_{\eta} \times A_{\eta}^{\prime}$ by $\mathbf{G}_{m}$ defines a perfect pairing (cf. [22, IX, 1.0.3]) (the Weil pairing):

$$
\langle\cdot, \cdot\rangle: U \otimes U^{\prime} \longrightarrow \mathbf{Z}_{\ell}(1) .
$$

The orthogonality theorem is the following formula $([22,2.4])$ :

$$
W=V \cap V^{\prime \perp},
$$

where $(\cdot)^{\perp}$ means the orthogonal for the pairing (3.2). Let $g$ be the dimension of $A_{\eta}, \mu$ be that of the torus $T$, and $\alpha$ (resp. $\lambda$ ) be the abelian (resp. unipotent) $\operatorname{rank}^{(11)}$ of $A_{s}^{0}$. By (3.3) we have $\operatorname{rk}\left(U /\left(V+V^{\prime \perp}\right)\right)=\operatorname{rk}(W)=\mu$, and $\operatorname{rk}\left(\left(V+V^{\prime \perp}\right) / V^{\prime \perp}\right)=\operatorname{rk}(V / W)=2 \alpha$, so, as $g=\alpha+\lambda+\mu$, we get

$$
\operatorname{rk}\left(V^{\prime \perp} / W\right)=2 \lambda \text {. }
$$

By definition, $A_{\eta}$ has semistable reduction over $S$ if and only if $\lambda=0$, which, by (3.4) is equivalent to $V^{\prime \perp} \subset V$.

From this it is immediate to prove Theorem 3.3. Indeed, as its action is unipotent, $I$ acts on $U$ (and $U^{\prime}$ ) through its tame quotient $I_{\mathrm{t}}=\widehat{\mathbf{Z}}^{\prime}(1)$ (and even through its $\ell$-primary part $\left.\mathbf{Z}_{\ell}(1)\right)$. We have to show $V^{\prime \perp} \subset V$, i.e., if $g$ is a topological generator of $I_{\mathrm{t}}$, that $g-1$ is zero on $V^{\prime \perp}$. But, by assumption, $(g-1)^{2}$ is zero on $U$, hence on $U^{\prime}$, hence $g-1$ is zero on $U^{\prime} / V^{\prime}$, hence on $\left(U^{\prime} / V^{\prime}\right)^{\vee}(1)$, but under $(3.2),\left(U^{\prime} / V^{\prime}\right)^{\vee}(1)=V^{\prime \perp}$.

The first appearance of (3.3) is in a paper of Igusa [32]. Igusa considers the case where $A_{\eta}$ is the Jacobian of $X_{\eta}$, for $X / S$ a proper curve with geometrically connected fibers and semistable reduction, smooth outside a unique rational point $x$ of $X_{s}$. He deduces (3.3) from what is called, in

(9) For $U / V$ this is because $U^{I}=\left(U \otimes \mathbf{Q}_{\ell}\right)^{I} \cap U$, for $V / W$ because over an algebraic closure of $s, A_{s}^{0} / T$ becomes an extension of an abelian variety by a unipotent group.

(10) The $\mathcal{E} \mathrm{xt}^{1}$ group is calculated in the category of abelian sheaves on the fppf site of $\eta$. This identification is classical: see [67, p. 196] for its history, and [24, XI, Th. 2.2] for a proof of a generalization over a locally noetherian base.

(11) i.e., the dimension of the quotient abelian variety (resp. unipotent part) of $A_{s}^{0} / T$ over an algebraic closure of $s$. 
today's language, a Picard-Lefschetz formula at $x$ for $\ell$-adic vanishing cycles. This inspired to Grothendieck his theory of the monodromy pairing, that we discuss in the next section. However, Grothendieck's proof of (3.3) does not involve any vanishing cycles. These are, somehow, replaced by the Néron models, and Grothendieck obtains (3.3) as a consequence of a vast theory of bi-extensions, developed in [22, VII, VIII], considerably generalizing and, should I say, simplifying - the notion initially introduced by Mumford for formal groups [52]. The inclusion $W \subset V \cap V^{\perp \perp}$ is more or less formal. The fact that it is an equality is proved in [22, IX 2.4] as a corollary of an ampleness criterion of Raynaud ([57, XI 1.11]).

Remarks 3.4.

(a) The converse of Theorem 3.3 holds: if $A_{\eta}$ has semistable reduction over $S$, then, for all $g \in I,(g-1)^{2}=0$ on $T_{\ell}\left(A_{\eta}\right)$. Indeed, as $V^{\perp}=\left(U^{\prime} / V^{\prime}\right)^{\vee}(1)$ is contained in $V=U^{I},(g-1)^{2}$ is zero on $U^{\prime}$, hence on $U$.

(b) There is a variant of Theorem 3.3 (and its converse) for the case of good reduction, namely $A_{\eta}$ has good reduction over $S$ (i.e., $A$ is an abelian scheme over $S)$ if and only if $I$ acts trivially on $T_{\ell}\left(A_{\eta}\right)$ ([22, IX 2.2.9]). This is the so-called Néron-Ogg-Shafarevich criterion for good reduction. The proof is easy. It does not use the orthogonality relation (3.3).

(c) In [22, IX 2.6 a)] Grothendieck observes that the inclusion $W \subset$ $V \cap V^{\perp \perp}$ can be proved by an arithmetic argument, independent of the theory of bi-extensions, using Weil's theorem on the weights of Frobenius for abelian varieties over finite fields. Pushing the argument further, Deligne was able to prove the semistable reduction theorem 3.2, bypassing (3.3) ([22, I 6]), and, in fact, getting it as a bonus (his argument gives $\left(\left(V \cap V^{\perp \perp}\right) / W\right) \otimes \mathbf{Q}_{\ell}=0$, hence (3.3), as $V / W$ is free over $\mathbf{Z}_{\ell}$, as recalled after (3.1)).

\section{Grothendieck's monodromy pairing}

4.1. Let $(S, s, \eta)$ and $A_{\eta}$ be as in Theorem 3.1. We denote by $A_{\eta}^{\prime}$ the dual abelian variety, by $A$ (resp. $A^{\prime}$ ) the Néron model of $A_{\eta}$ (resp. $A_{\eta}^{\prime}$ ), and by $T$ (resp. $T^{\prime}$ ) the maximal subtorus of $A_{s}^{0}\left(\right.$ resp. $\left.A_{s}^{\prime 0}\right)$. From now on - unless otherwise stated - we assume that $A_{\eta}$ has semistable reduction over $S$, i.e., $A_{s}^{0}$ is extension of an abelian variety $B$ by the torus $T$. It follows from the criterion 3.3 that $A_{\eta}^{\prime}$ also has semistable reduction, i.e., $A_{s}^{\prime 0}$ is extension of an abelian variety $B^{\prime}$ by $T^{\prime}$. 
As in the previous section, we consider the Tate modules $U=T_{\ell}\left(A_{\eta}\right)$, $U^{\prime}=T_{\ell}\left(A_{\eta}^{\prime}\right)$, and their 2-step filtrations $(W \subset V \subset U),\left(W^{\prime} \subset V^{\prime} \subset\right.$ $U^{\prime}$ ), where $V=U^{I}=T_{\ell}\left(A_{s}^{0}\right), W=T_{\ell}(T)$, and similarly $V^{\prime}=U^{\prime I}=$ $T_{\ell}\left(A_{s}^{\prime 0}\right), W^{\prime}=T_{\ell}\left(T^{\prime}\right)$. Following Grothendieck's notation, we denote by $M$ the character group of $T^{\prime}$ :

$$
M=\mathcal{H o m}\left(T^{\prime}, \mathbf{G}_{m}\right),
$$

so that the co-character group is $M^{\vee}=\mathcal{H} \mathrm{om}\left(\mathbf{G}_{m}, T^{\prime}\right)=\mathcal{H} \mathrm{om}(M, \mathbf{Z})$ (these are free finitely generated $\mathbf{Z}$-modules with action of $\operatorname{Gal}(\widetilde{s} / s)$ (in the notation (1.1)), and similarly

$$
M^{\prime}=\mathcal{H o m}\left(T, \mathbf{G}_{m}\right),
$$

with $M^{\prime \vee}=\mathcal{H o m}\left(\mathbf{G}_{m}, T\right)=\mathcal{H o m}\left(M^{\prime}, \mathbf{Z}\right)$. As $T^{\prime}\left[\ell^{n}\right]=\mathcal{H} \operatorname{om}\left(\mathbf{Z} / \ell^{n}, T^{\prime}\right)=$ $\mathcal{H} \mathrm{om}\left(\mu_{\ell^{n}}, T^{\prime}\right) \otimes \mu_{\ell^{n}}=\left(M^{\vee} / \ell^{n} M^{\vee}\right) \otimes \mu_{\ell^{n}}$, we have

$$
W^{\prime}=M^{\vee} \otimes \mathbf{Z}_{\ell}(1),
$$

and similarly

$$
W=M^{\prime \vee} \otimes \mathbf{Z}_{\ell}(1) .
$$

Let $M_{\ell}:=M \otimes \mathbf{Z}_{\ell}, M_{\ell}^{\prime}:=M^{\prime} \otimes \mathbf{Z}_{\ell}$. As $A_{\eta}$ has semistable reduction, by (3.3) and (3.4), we have

$$
W=V^{\prime \perp}=\left(U^{\prime} / V^{\prime}\right)^{\vee}(1),
$$

hence by (4.4),

$$
U^{\prime} / V^{\prime}=M_{\ell}^{\prime}
$$

and similarly

$$
U / V=M_{\ell},
$$

which formulas are probably the reason for the a priori strange notation (4.1).

As $I$ acts on $U$ through its quotient $t_{\ell}: I \rightarrow \mathbf{Z}_{\ell}(1)$, and unipotently of exponent $\leqslant 1$, there exists a unique homomorphism (the monodromy operator)

$$
N: U \longrightarrow U(-1)
$$

such that $g x=x+t_{\ell}(g) N x$ for all $x \in U$ and $g \in I$. We have $N^{2}=0$, and $N$ is $\operatorname{Gal}(\bar{\eta} / \eta)$-equivariant. We again denote by $N: U^{\prime} \rightarrow U^{\prime}(-1)$ the monodromy operator corresponding to $A_{\eta}^{\prime}$. By definition, $V$ and $V^{\prime}$ are the kernels of $N$. As the Weil pairing (3.2) is Galois equivariant, in particular, $I$-equivariant, it satisfies the formula

$$
\langle N x, y\rangle+\langle x, N y\rangle=0 .
$$

This implies that $N(U) \subset V^{\prime \perp}(-1)(=W(-1)$ by $(4.5))$, and, as $N(V)=0$, $N$ induces a homomorphism

$$
\begin{gathered}
N: U / V \longrightarrow W(-1), \\
-94-
\end{gathered}
$$


which, by (4.5) and (4.6), can be rewritten

$$
u_{\ell}: M_{\ell} \longrightarrow M_{\ell}^{\prime \vee}
$$

or, equivalently, a pairing

$$
\langle\cdot, \cdot\rangle_{\ell}: M_{\ell} \otimes M_{\ell}^{\prime} \longrightarrow \mathbf{Z}_{\ell} .
$$

This is Grothendieck's monodromy pairing. By definition, $u_{\ell}$ is injective, hence is an isogeny. The pairing (4.11) is symmetric, i.e., the pairing deduced by exchanging $A_{\eta}$ and $A_{\eta}^{\prime}$ (and identifying $A_{\eta}$ with $\left(A_{\eta}^{\prime}\right)^{\prime}$ by the biduality isomorphism) is obtained from (4.11) by $\langle x, y\rangle \mapsto\langle y, x\rangle$. Grothendieck's main result is the following ([22, IX 10.4]) (discussed for the first time in a letter to Serre, dated October 3-5, 1964, see [15, p. 207, 209]):

THEOREM 4.1.

(a) There exists a unique homomorphism

$$
u: M \longrightarrow M^{\prime \vee}
$$

such that $u \otimes \mathbf{Z}_{\ell}=u_{\ell}$ for all $\ell$. Let

$$
\langle\cdot, \cdot\rangle: M \otimes M^{\prime} \longrightarrow \mathbf{Z}
$$

denote the pairing defined by (4.12).

(b) Let $\xi: A_{\eta} \rightarrow A_{\eta}^{\prime}$ be a polarization, $\xi^{*}: M \rightarrow M^{\prime}$ the homomorphism deduced by functoriality. Then the pairing

$$
\langle\cdot, \cdot\rangle_{\xi}: M \otimes M \longrightarrow \mathbf{Z}
$$

defined by $\langle x, y\rangle_{\xi}=\left\langle x, \xi^{*} y\right\rangle$ is symmetric, and negative definite ${ }^{(12)}$.

We will sketch Grothendieck's proof at the end of Section 4. An alternate construction of $u$ and proof of (Theorem 4.1(b)) was given by Raynaud, using rigid methods ([59] and [22, IX 14]).

Remark 4.2. - The construction of $u_{\ell}$ (4.10) makes essential use of the hypothesis $\ell \neq p$. Let $M_{p}:=M \otimes \mathbf{Z}_{p}, M_{p}^{\prime}:=M^{\prime} \otimes \mathbf{Z}_{p}$. For $S$ of mixed characteristic, using Tate's theorem on homomorphisms of Barsotti-Tate groups ([73, Th. 4]), Grothendieck directly constructs in ([22, IX 9]) a homomorphism $u_{p}: M_{p} \rightarrow M_{p}^{\prime \vee}$ in terms of the pro- $p$-groups $T_{p}\left(A^{0}\right), T_{p}\left(A^{\prime 0}\right)$, and, by an analytic argument, shows (in [22, IX 12]) that (Theorem 4.1(a)) extends to $\ell=p$, i.e., $u_{p}=u \otimes \mathbf{Z}_{p}$. Now that thanks to de Jong [44] Tate's theorem has been established in equal characteristic, the restriction on $S$ is superfluous $^{(13)}$.

(12) In [22, IX, X 4], it is asserted to be positive definite. This discrepancy seems to be due to a sign in the Picard-Lefschetz formula.

(13) A construction of $u_{p}$ valid without assuming $S$ of mixed characteristic, and using only Tate's theorem (but over a higher dimensional normal base, a formal moduli scheme as in [22, IX 12.8]), has been made by B. Conrad (private communication). 
In the sequel, we assume again $\ell \neq p$. An immediate consequence of Theorem 4.1, actually just the fact that $u_{\ell}$ is an isogeny, is:

Corollary 4.3.

(a) Consider the filtration $(W \subset V \subset U)(3.1)$ as an increasing filtration $\left(M_{i}\right)_{i \in \mathbf{Z}}$, with $M_{i}=U$ for $i \geqslant 1, M_{0}=V, M_{-1}=W, M_{i}=0$ for $i \leqslant-2$. Then $M_{i} \otimes \mathbf{Q}_{\ell}$ is the monodromy filtration of $U \otimes$ $\mathbf{Q}_{\ell}$ with respect to the nilpotent operator $N$, i.e., $N\left(M_{i} \otimes \mathbf{Q}_{\ell}\right) \subset$ $M_{i-2} \otimes \mathbf{Q}_{\ell}(-1)$, and $N^{i}: \operatorname{gr}_{i}^{M}\left(U \otimes \mathbf{Q}_{\ell}\right) \rightarrow \operatorname{gr}_{-i}^{M}\left(U \otimes \mathbf{Q}_{\ell}\right)(-i)$ is an isomorphism for all $i \geqslant 0$.

(b) Assume that $k$ is a finite field $\mathbf{F}_{q}$. Then the filtration $\left(M_{i}\right)$ is pure, i.e., equals, up to a shift, the weight filtration of $U \otimes \mathbf{Q}_{\ell}$ in the sense of Deligne $([21,1.7 .5]): \operatorname{gr}_{i}^{M} U$ is pure ${ }^{(14)}$ of weight $i-1$.

As $H^{1}\left(A_{\bar{\eta}}\right):=H^{1}\left(A_{\bar{\eta}}, \mathbf{Z}_{\ell}\right)$ is dual to $U=T_{\ell}\left(A_{\eta}\right)$, the filtration, still denoted $M_{\bullet}$ on $H^{1}\left(A_{\bar{\eta}}\right)$ dual to the filtration $M_{\bullet}$ on $U$, is again the monodromy filtration (for the monodromy operator $N$ ), and when $k=\mathbf{F}_{q}$, is the weight filtration up to shift : $\operatorname{gr}_{i}^{M} H^{1}\left(A_{\bar{\eta}}\right)$ is pure of weight $i+1$.

As we mentioned in Remark 3.4(c), Deligne gave an alternate short proof of this. However, weight arguments don't work for the next corollary 4.4, which lies deeper. We need some preliminary remarks before stating it.

4.2. For the moment, we don't assume that $A_{\eta}$ has semistable reduction over $S$. Consider the finite commutative étale group scheme over $s$ of connected components of the special fiber of the Néron model $A$ of $A_{\eta}$,

$$
\Phi_{0}:=\Phi_{0}\left(A_{\eta}\right):=A_{s} / A_{s}^{0},
$$

and similarly define $\Phi_{0}^{\prime}:=\Phi_{0}\left(A_{\eta}^{\prime}\right):=A_{s}^{\prime} / A_{s}^{\prime 0}$. In [22, IX], Grothendieck :

(i) defined a canonical pairing $\Phi_{0} \times \Phi_{0}^{\prime} \rightarrow \mathbf{Q} / \mathbf{Z}$, which he conjectured to be perfect;

(ii) in the semistable reduction case, constructed a canonical isomorphism between $\Phi_{0}$ and the cokernel of $u: M \rightarrow M^{\prime \vee}$ (4.12).

Let me first discuss (ii). We now assume that $A_{\eta}$ has semistable reduction. For simplicity, assume that $S$ is strictly local (so that $S=\widetilde{S}$ in the notation (1.1), and $\Phi_{0}, \Phi_{0}^{\prime}$ are usual finite groups), and take $\ell \neq p$. Let $\Phi_{0}(\ell)$ be the $\ell$-primary component of $\Phi_{0}$. The $\ell$-primary component of Coker $(u)$ is

$$
\operatorname{Coker}(u)(\ell)=\operatorname{Ker}\left(u_{\ell} \otimes \mathbf{Q}_{\ell} / \mathbf{Z}_{\ell}: M_{\ell} \otimes \mathbf{Q}_{\ell} / \mathbf{Z}_{\ell} \rightarrow M_{\ell}^{\prime \vee} \otimes \mathbf{Q}_{\ell} / \mathbf{Z}_{\ell}\right) .
$$

(14) i.e., the eigenvalues of the geometric Frobenius $F$ are $q$-Weil numbers of weight $i-1$; moreover (by Weil), $\operatorname{det}\left(1-F t, \operatorname{gr}_{i}^{M}\left(U \otimes \mathbf{Q}_{\ell}\right)\right)$ has coefficients in $\mathbf{Z}$ and is independent of $\ell$. 
On the other hand, as $A_{s}^{0}$ is $\ell$-divisible, we have

$$
\Phi_{0}(\ell)=A_{s}(\ell) / A_{s}^{0}(\ell)
$$

where $(\cdot)(\ell)=\underline{\lim }(\cdot)\left[\ell^{n}\right]$, hence

$$
\Phi_{0}(\ell)=\left(U \otimes \mathbf{Q}_{\ell} / \mathbf{Z}_{\ell}\right)^{I} /\left(V \otimes \mathbf{Q}_{\ell} / \mathbf{Z}_{\ell}\right),
$$

with the above notation $U=T_{\ell}\left(A_{\eta}\right), V=U^{I}=T_{\ell}\left(A_{s}^{0}\right)^{(15)}$. We thus have an injection

$$
\Phi_{0}(\ell) \hookrightarrow(U / V) \otimes \mathbf{Q}_{\ell} / \mathbf{Z}_{\ell} \stackrel{(4.7)}{=} M \otimes \mathbf{Q}_{\ell} / \mathbf{Z}_{\ell}
$$

Now using that $A_{\eta}$ has semistable reduction, Grothendieck extends the definition of (4.16) to $\ell=p$, and proves:

COROLlary 4.4. - For $A_{\eta}$ having semistable reduction, the homomorphism (4.16) induces a short exact sequence

$$
0 \longrightarrow \Phi_{0}(\ell) \longrightarrow M \otimes \mathbf{Q}_{\ell} / \mathbf{Z}_{\ell} \stackrel{u \otimes \mathbf{Q}_{\ell} / \mathbf{Z}_{\ell}}{\longrightarrow} M^{\prime \vee} \otimes \mathbf{Q}_{\ell} / \mathbf{Z}_{\ell} \longrightarrow 0
$$

for all primes $\ell$, where $u$ is the homomorphism (4.12). In other words, the maps (4.17) induce an isomorphism

$$
\Phi_{0} \stackrel{\sim}{\longrightarrow} \operatorname{Coker}\left(u: M \rightarrow M^{\prime \vee}\right) .
$$

The proof for $\ell \neq p$ is easy: from (4.15) a simple calculation shows that $\Phi_{0}(\ell)$ is the torsion subgroup of $H^{1}(I, U)=U / N U(1)([22, \operatorname{IX}(11.3 .8)])$, i.e., $W / N U(1)$, which, by (4.5) and the definition of $u_{\ell}$ is just $\operatorname{Coker}\left(u_{\ell}\right)$. The proof for $\ell=p$ is more delicate.

Remark 4.5. - When $A_{\eta}$ is the Jacobian of the generic fiber of a proper, flat curve $X / S$, with geometrically connected fibers and semistable reduction (in other words, $X$ is regular, $X_{\eta}$ is smooth, and $X_{s}$ is a (reduced) divisor with normal crossings), i.e., $A_{\eta}=\operatorname{Pic}_{X_{\eta} / \eta}^{0}$ (see e.g. $[14,9.2]$ ) for general properties of Jacobians), (4.18) leads (via the Picard-Lefschetz formula) to a purely combinatorial description of $\Phi_{0}$, in terms of the irreducible components and double points of the special fiber $X_{s}$. We will briefly discuss this in 6.1 and 6.3. Similar descriptions of $\Phi_{0}$ under milder assumptions on $X$ were given independently by Raynaud [58], see also [14, 9.6], [33].

An interesting special case computed by Deligne, and discussed by MazurRapoport in [49] is when $S=\operatorname{Spec} \mathbf{Z}_{p}, X$ is the modular curve $X_{0}(p)$ localized over $S$, with $p \geqslant 5$. Then, over an algebraic closure of $\mathbf{F}_{p}, \Phi_{p}:=\Phi_{0}\left(J_{p}\right)$ ( $J_{p}$ the special fiber of the Néron model) is a cyclic group of order the numerator of $(p-1) / 12([49, \mathrm{~A} 1])$, having as a generator the image of the Q-rational divisor $(0)-(\infty)$. As an application, Mazur shows that, for $\ell \neq p$, the Hecke operator $T_{\ell}$ on $\Phi_{p}$ equals $1+\ell([49,9.7])$. Ribet proved, more generally, that

(15) This formula does not use that $A_{\eta}$ has semistable reduction. 
$T_{\ell}=1+\ell$ on $\Phi_{0}\left(J_{p}\left(X_{0}(p N)\right)\right)($ for $(\ell, p N)=1,(p, N)=1)([60,(3.12)])$, a result he used to prove that the Shimura-Taniyama-Weil conjecture implies Fermat.

4.3. Let us recall the definition of the pairing mentioned in 4.2 (i). Let

$$
w_{\eta} \in \operatorname{Ext}^{1}\left(A_{\eta} \otimes^{L} A_{\eta}^{\prime}, \mathbf{G}_{m}\right)
$$

be the Poincaré bi-extension (tensor product taken over Z) (see [22, VII 2.9.5, VIII 3.2], where reference [1] is [24] of the present paper) and consider the immersions $i: s \hookrightarrow S, j: \eta \hookrightarrow S$. As $R^{1} j_{*} \mathbf{G}_{m}=0$ (Hilbert 90), the exact sequence (of group schemes over $S$ )

$$
0 \longrightarrow \mathbf{G}_{m} \longrightarrow j_{*} \mathbf{G}_{m} \longrightarrow i_{*} \mathbf{Z} \longrightarrow 0
$$

(where the middle term is the Néron-Raynaud model of $\mathbf{G}_{m}$, a smooth commutative group scheme over $S$ ) yields an exact sequence

$$
\operatorname{Ext}^{1}\left(A \otimes{ }^{L} A^{\prime}, \mathbf{G}_{m}\right) \longrightarrow \operatorname{Ext}^{1}\left(A_{\eta} \otimes^{L} A_{\eta}^{\prime}, \mathbf{G}_{m}\right) \stackrel{c}{\longrightarrow} \operatorname{Ext}^{1}\left(A \otimes{ }^{L} A^{\prime}, i_{*} \mathbf{Z}\right) .
$$

On the other hand, a boundary map gives a canonical isomorphism

$$
\delta: \operatorname{Hom}\left(\Phi_{0} \otimes \Phi_{0}^{\prime}, \mathbf{Q} / \mathbf{Z}\right) \stackrel{\sim}{\longrightarrow} \operatorname{Ext}^{1}\left(A \otimes^{L} A^{\prime}, i_{*} \mathbf{Z}\right) .
$$

Grothendieck defines the pairing ([22, IX (1.2.1)])

$$
w_{0}: \Phi_{0} \otimes \Phi_{0}^{\prime} \longrightarrow \mathbf{Q} / \mathbf{Z}
$$

as the image of $w_{\eta}(4.19)$ by $\delta^{-1} c$. This is the obstruction to extending $w_{\eta}$ to a bi-extension $w \in \operatorname{Ext}^{1}\left(A \otimes^{L} A^{\prime}, \mathbf{G}_{m}\right)$. Grothendieck conjectured that the pairing $w_{0}$ is perfect. Here is a brief history of the question:

- In the semistable reduction case, Corollary 4.4 gives the existence of a perfect pairing $w_{0}^{\prime}$ of the form (4.23) and Grothendieck conjectured that, up to a sign that should be determined, it coincides with $w_{0}$ $\left([22, \text { IX 11.4] })^{(16)}\right.$.

- Various cases were treated by Bégueri [4], McCallum [50], Bosch [12], Bosch-Lorenzini [13].

- Counter-examples for $k$ not perfect were given by BertapelleBosch [9], using Weil restrictions, and by Bosch-Lorenzini [13] for Jacobians.

- A proof in the general case ( $k$ perfect) was given by Suzuki [72] (see also [71] for a generalization).

(16) The definition of a perfect pairing of the form $w_{0}$ on the $\ell$-primary components, $\ell \neq p$, using (4.15) is easy ([22, IX 11.3]) and doesn't need the semi-stability assumption; its coincidence with $w_{0}$ was checked by Bertapelle [8]. In the semistable case, the verification of the coincidence between $w_{0}^{\prime}$ and $w_{0}$ was made by Werner [76], using the rigid geometry of Raynaud's extensions in [59]. 
While in the works of Bertapelle, Bosch, Bosch-Lorenzini, Werner, the main tools are those provided by the geometry of abelian varieties, Bégueri's approach exploits another ingredient, namely Serre's geometric local class field theory, the perfection of Grothendieck's pairing appearing as a byproduct of the reciprocity isomorphism. It seems to me, however, that the relation between these various methods is not yet fully understood. For example, in the case of the Jacobian of a proper, smooth curve having semistable reduction, the pairing (4.11), as described below by (4.37) via Picard-Lefschetz, should be more directly related to Néron's height pairing: one would like to exhibit the vanishing cycles hidden in [13, 4.4].

4.4. We now sketch Grothendieck's proof of Theorem 4.1. In Theorem 4.1 (a) the uniqueness of $u$ is clear (one $\ell$ even suffices). The construction of $u$ is easily reduced to the case where $A_{\eta}$ is the Jacobian of a proper smooth and geometrically irreducible curve $X_{\eta} / \eta$ ([22, IX 10.5.1-10.5.3]). At this point, Grothendieck uses a result that was proved at about the same time by Deligne and Mumford ${ }^{(17)}[23]$ as a corollary to Theorem 3.2, the so-called semistable reduction theorem for curves, thanks to which, after a finite extension of $\eta, X_{\eta}$ admits a proper, flat model $X / S$ with $X$ regular, having semistable reduction, i.e. étale locally étale over $S[x, y] /(x y-t)$, where $t$ is a uniformizing parameter of $S$. Thus, after a further reduction, we may - and we will in the following - assume that $X_{\eta}$ is the generic fiber of a model $X / S$ as above, and also that the residue field $k$ is separably closed. In this situation, we have combinatorial descriptions of $M$ and $u_{\ell}$ in terms of the dual graph of $X_{s}$, and they suggest the definition of $u$.

(a) Description of $M$. - By the canonical polarization of $A_{\eta}=\operatorname{Pic}_{X_{\eta} / \eta}^{0}$, we identify $A_{\eta}$ and $A_{\eta}^{\prime}$, hence their Néron models $A$ and $A^{\prime}$, the maximal tori $T$ and $T^{\prime}$ in their special fibers, and their character groups $M$ and $M^{\prime}$. Thus, by (4.1), $M^{\vee}$ is the co-character group of $T$. On the other hand, by a result of Raynaud, $A_{s}^{0}=\operatorname{Pic}_{X_{s} / s}^{0}$ ([22, IX (12.1.12)]). Put $Y:=X_{s}$, denote by $\Gamma(Y)$ its dual graph. A simple calculation ([22, IX 12.3], [33, 2.3]) shows that

$$
M=H_{1}(\Gamma(Y), \mathbf{Z})
$$

(and $M^{\vee}=H^{1}(\Gamma(Y), \mathbf{Z})$ ). More explicitly, if $J$ is the set of irreducible components of $Y, \Sigma$ the set of double points of $Y$, and, if for each $x \in \Sigma$ we choose an order on the set $J_{x}$ of the two branches passing through $x$ (points $x_{1}, x_{2}$ of the normalization of $Y$ sitting over $x$ ), so that we have a basis $\delta^{\prime}(x)=(1,-1)$ of the kernel $\mathbf{Z}^{\prime}(x)$ of the sum map $\mathbf{Z}^{J_{x}} \rightarrow \mathbf{Z}$, then we have

(17) Other proofs, independent of Theorem 3.2, were found later: Artin-Winters [3], T. Saito [61], Temkin [74]. 
an exact sequence

$$
0 \longrightarrow M \longrightarrow \bigoplus_{x \in \Sigma} \mathbf{Z}^{\prime}(x) \longrightarrow \mathbf{Z}^{J}
$$

where $J_{x}=\left(x_{1}, x_{2}\right)$ and the second map sends $\delta^{\prime}(x)$ to the difference $C_{x_{1}}-$ $C_{x_{2}}$ of the components of $Y$ corresponding to $x_{1}$ and $x_{2}$. Dually ${ }^{(18)}$, we have an exact sequence

$$
\mathbf{Z}^{J} \longrightarrow \bigoplus_{x \in \Sigma} \mathbf{Z}(x) \longrightarrow M^{\vee} \longrightarrow 0
$$

where $\mathbf{Z}(x)$ is the cokernel of the diagonal map $\mathbf{Z} \rightarrow \mathbf{Z}^{J_{x}}$, with basis $\delta(x)$ dual to $\delta^{\prime}(x)$, and the first map is dual to the second map in (4.25).

(b) Description of $N$. - The specialization sequence (1.8) for $K=\mathbf{Z}_{\ell}(1)$ gives an exact sequence

$$
\begin{aligned}
0 \longrightarrow H^{1}\left(X_{s}\right)(1) \longrightarrow H^{1}\left(X_{\bar{\eta}}\right) & (1) \longrightarrow \Phi^{1}(1) \\
& \longrightarrow H^{2}\left(X_{s}\right)(1) \longrightarrow H^{2}\left(X_{\bar{\eta}}\right)(1) \longrightarrow 0,
\end{aligned}
$$

where $H^{i}(\cdot)=H^{i}\left(\cdot, \mathbf{Z}_{\ell}\right), \Phi^{i}:=H^{i}\left(X_{s}, R \Phi\left(\mathbf{Z}_{\ell}\right)\right)$. The vanishing cycles groups $R^{q} \Phi\left(\mathbf{Z}_{\ell}\right)$ were calculated by Deligne in ([22, XV 3]) (as a special case of pencils with ordinary quadratic singularities). The complex $R \Phi\left(\mathbf{Z}_{\ell}\right)$ is concentrated in degree 1 and on the set $\Sigma$ of double points of $X_{s}$, so that

$$
\Phi^{1}(1)=\bigoplus_{x \in \Sigma} \Phi^{1}(1)_{x}
$$

and, with the choice of the ordering on $J_{x}$ made above, $\Phi^{1}(1)_{x}$ has a distinguished basis $\delta_{x}^{\prime}$, called the vanishing cycle at $x$ :

$$
\Phi^{1}(1)_{x}=\mathbf{Z}_{\ell} \delta_{x}^{\prime} .
$$

By definition (4.7), $M_{\ell}=T_{\ell}\left(A_{\eta}\right) / T_{\ell}\left(A_{s}^{0}\right)=H^{1}\left(X_{\bar{\eta}}\right)(1) / H^{1}\left(X_{s}\right)(1)$, hence, by (4.27),

$$
M_{\ell}=\operatorname{Ker}\left(\Phi^{1}(1) \rightarrow H^{2}\left(X_{s}\right)(1)\right) .
$$

Dually to (4.28) and (4.29), we have

$$
H_{\Sigma}^{1}\left(X_{s}, R \Psi\left(\mathbf{Z}_{\ell}\right)\right)=\bigoplus_{x \in \Sigma} H_{x}^{1}\left(X_{s}, R \Psi\left(\mathbf{Z}_{\ell}\right)\right),
$$

with $H_{x}^{1}\left(X_{s}, R \Psi\left(\mathbf{Z}_{\ell}\right)\right.$ ) dual to $\Phi_{x}^{1}(1)$ (with values in $\mathbf{Z}_{\ell}$ ), with dual basis $\delta_{x}$ :

$$
H_{x}^{1}\left(X_{s}, R \Psi\left(\mathbf{Z}_{\ell}\right)\right)=\mathbf{Z}_{\ell} \delta_{x}
$$

(18) The cokernel of the last map is $\mathbf{Z}$, as $Y$ is geometrically connected, hence $\Gamma(Y)$ connected. 
The monodromy operator $N$ on $U=T_{\ell}\left(A_{\bar{\eta}}\right)=H^{1}\left(X_{\bar{\eta}}\right)$ factors (by definition of $R \Phi)$ through a sum of local variation maps

$$
N_{x}: \Phi_{x}^{1}(1)=\mathbf{Z}_{\ell} \cdot \delta_{x}^{\prime} \longrightarrow H_{x}^{1}\left(X_{s}, R \Psi\left(\mathbf{Z}_{\ell}\right)\right)=\mathbf{Z}_{\ell} \cdot \delta_{x},
$$

i.e., we have a commutative diagram

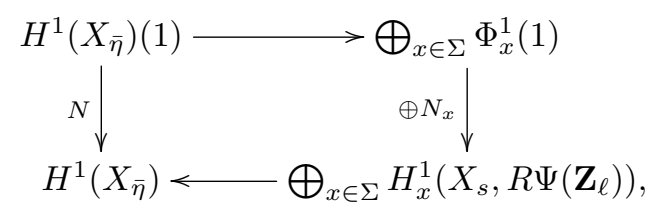

where $N_{x}$ is an isomorphism, the Picard-Lefschetz isomorphism (see 6.1), given by

$$
N_{x}\left(\delta_{x}^{\prime}\right)=-\delta_{x}
$$

Using duality and the cospecialization sequence, dual to (4.27),

$$
H^{1}\left(X_{\bar{\eta}}\right) \longleftarrow H_{\Sigma}^{1}(R \Psi) \longleftarrow H^{0}\left(\widetilde{X}_{s}\right) \longleftarrow H^{0}\left(X_{\bar{\eta}}\right) \longleftarrow 0,
$$

where $\widetilde{X}_{s}$ is the normalization of $X_{s}$, one checks that the factorization (4.34) is refined into the following one:

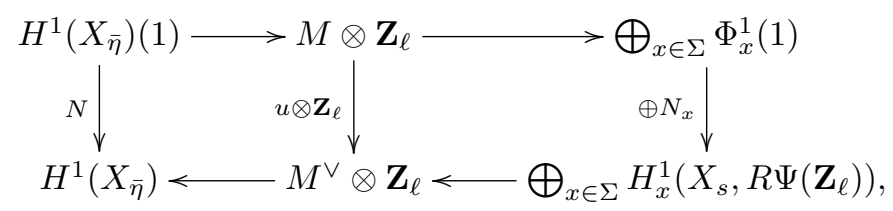

in which $u: M \rightarrow M^{\vee}$ is the map making the following square commute:

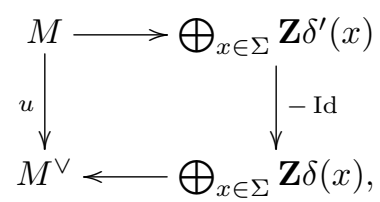

where the upper (resp. lower) row is the injection (4.25) (resp. surjection (4.26)). In other words, $u$ is induced by the negative definite quadratic form $\sum-t_{i}^{2}$ on $\mathbf{Z}^{r}=\sum_{x \in \Sigma} \mathbf{Z}$.

In the case considered by Igusa [32] — which was for Grothendieck the starting point of the whole theory - the special fiber $X_{s}$ is irreducible, and $\Sigma$ consists of a single point $x$. The rows of (4.38) are isomorphisms (hence $u$ also). If we identify $\delta_{x}$ with its image in $M^{\vee} \otimes \mathbf{Z}_{\ell} \subset H^{1}\left(X_{s}\right) \subset H^{1}\left(X_{\bar{\eta}}\right)$ by the bottom arrow of $(4.37),(4.27)$ yields a short exact sequence

$$
0 \longrightarrow H^{1}\left(X_{s}\right)(1) \longrightarrow H^{1}\left(X_{\bar{\eta}}\right)(1) \stackrel{N}{\longrightarrow} \mathbf{Z}_{\ell} \delta_{x} \longrightarrow 0,
$$


where $H^{1}\left(X_{s}\right)$ is the fixed part, and $\mathbf{Z}_{\ell} \delta_{x}$ the toric part

$$
M^{\vee} \otimes \mathbf{Z}_{\ell}=W(-1)=T_{\ell}(T)(-1)=H^{1}\left(X_{s}\right)(1)^{\perp},
$$

where $T$ is the maximal torus in $A_{s}^{0}$, i.e., the 1-dimensional torus at $x$ defined by $\pi_{*} \mathbf{G}_{m} / \mathbf{G}_{m}, \pi: \widetilde{X}_{s} \rightarrow X_{s}$ being the normalization map. Elements $\sigma$ of the inertia group $I$ act on $H^{1}\left(X_{\bar{\eta}}\right)(1)$ by the symplectic transvections $a \mapsto a-t_{\ell}(\sigma)\left\langle a, \delta_{x}\right\rangle \delta_{x}([22, \mathrm{XV} 3.4])$.

More generally, under the assumptions of 4.4 (b) on $X / S$, if $X_{s}$ is irreducible, then the map on $H^{2}$ in (4.27) is an isomorphism, and (4.34) yields an exact sequence similar to (4.39), with $\mathbf{Z}_{\ell} \delta_{x}$ replaced by $\bigoplus_{x \in \Sigma} \mathbf{Z}_{\ell} \delta_{x}$, which is again the toric part $M^{\vee} \otimes \mathbf{Z}_{\ell}$.

\section{Grothendieck's dreams}

In [22, IX, Introduction], Grothendieck writes:

“... le présent exposé peut aussi être considéré comme une étude dé-

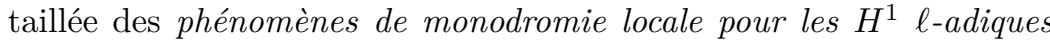
(ou mieux encore, pour les $H^{1}$ « motiviques») des variétés projectives et lisses sur $K$. Dans cette optique, il semble clair que les principaux résultats du présent exposé sont destinés à être englobés dans une « théorie de Néron » pour des motifs de poids quelconque, i.e. pour des $H^{i}$ ( $\ell$-adiques, ou de de Rham, ou de Hodge, etc.) avec $i$ quelconque, qu'on ne commence qu'à entrevoir à l'heure actuelle. (Cf. à ce sujet $[\mathrm{P}$. A. Griffiths, Report on variation of Hodge structures, à paraître ${ }^{(19)}$, et plus particulièrement les conjectures de Deligne 9.8 à 9.13 du rapport cité.)"

These questions have been at the origin of several vast theories:

- mixed Hodge theory

- theory of weights in $\ell$-adic cohomology

- $p$-adic Hodge theory

- mixed motives.

Hodge theory came first, with Deligne's fundamental work ([16], [17], [19]), and subsequent developments on the analytic and algebraic theory of variations of Hodge structures by Griffiths, Schmid, and many others. Grothendieck's conjectural "yoga" of weights over finite fields had inspired

(19) See [28]. See also [29], Deligne's report [16], and Griffiths-Schmid's survey [30], giving the state of the art in 1975, taking into account Deligne's work on Hodge theory. 
Deligne for his mixed Hodge theory. In turn, mixed Hodge theory gave some guidelines in his theory of mixed $\ell$-adic sheaves in [21].

At the end of [22, IX, Introduction], Grothendieck observes that, in the notation of Theorem 4.1, the Galois module $T_{p}\left(A_{\eta}\right)$ ( $p$ the residual characteristic) behaves quite differently from its $\ell$-adic analogue, $\ell \neq p$ (as indeed Tate's seminal article [73] had shown). He adds that Barsotti-Tate groups over $\eta$ play the role of $p$-adic local systems over $\eta$, those which appear in the $p$-adic analysis of the $H^{1}$ of projective, smooth varieties over $\eta$. He suggests that in order to understand the higher $H^{i}$ 's from a $p$-adic viewpoint, the category of Barsotti-Tate groups should be suitably enlarged, using inputs from crystalline cohomology, this new theory he had just introduced. This can be seen as the origin of $p$-adic Hodge theory, which really started only a couple of years later with Fontaine's foundational work on Grothendieck's problem of the mysterious functor.

The degenerating abelian varieties studied in [22, IX] are the prototype of mixed motives (over $S$, in cohomological degree 1). Grothendieck's dream of generalizing this theory to higher degree is far from being fulfilled today, even in the case of a base field, despite extensive work during the past forty years (starting with Deligne's theory of 1-motives in [19], up to the recent achievements accomplished by Voevodsky and many others, for which even a rough survey would by far exceed the scope of this report).

I will limit myself to a very brief update on $\ell$-adic vanishing cycles and monodromy.

\section{Update on the $\ell$-adic side}

6.1. Picard-Lefschetz. The Picard-Lefschetz formula in $\ell$-adic cohomology proved by Deligne in $[22, \mathrm{XV}]$ - and used by Grothendieck in the proof of Theorem 4.1 - was the key tool in the cohomological theory of Lefschetz pencils, developed in [22, XVIII], which provided the basic framework for Deligne's first proof of the Weil conjecture [18]. In the odd relative dimension $n$ case (and already for $n=1$, in which case Deligne's calculation in $[22, \mathrm{XV}]$ showed that (2.5) holds unconditionally), the proof given in [22, $\mathrm{XV} 3.3]$ is of transcendental nature, using a lifting to characteristic zero, a comparison theorem [22, XIV] between $\ell$-adic and Betti nearby cycles, and an explicit topological calculation in the Betti case. A purely algebraic proof was found later [36], as a by-product of Rapoport-Zink's description of the monodromy operator $N$ in the semistable reduction case (see 6.3). 
6.2. Structure of $R \Psi$. Let $S$ and $\Lambda$ be as in 1.1, and let $X$ be of finite type over $S$. Deligne proved in [20, Th. finitude] that for $K \in D_{c}^{b}\left(X_{\eta}\right)$, we have $R \Psi_{X}(K) \in D_{c}^{b}\left(X_{\bar{s}}\right)$. Finer results were obtained later in relation with the theory of perverse sheaves. It was proved in [7] that the functor $R \Psi$ is right t-exact (see the appendix in [7] for an alternate proof). Combined with a result of Gabber to the effect that $R \Psi$ commutes with duality, it implies that $R \Psi$ is t-exact, and in particular transforms perverse sheaves into perverse sheaves. Moreover, it was also proved by Gabber that, for $K$ perverse on $X, R \Phi_{X}(K)[-1]$ is perverse, and that $R \Psi$ commutes with external products (see [6], [34]). It was proved by Beilinson [48] that $R \Phi$ commutes with duality up to a twist. A new proof and generalizations over higher dimensional bases (cf. 6.6) are given in [47].

6.3. The semistable case. Assume that $X / S$ has semistable reduction: $X$ is regular, $X_{\eta}$ is smooth, and $X_{s}$ is a reduced divisor with normal crossings in $X$, i.e., étale locally at any geometric point $\bar{x}$ of $X_{s}, X$ is defined by an equation of the form $\prod_{1 \leqslant i \leqslant r} t_{i}=\pi$, where $\pi$ is a uniformizing parameter of $S$ and the $t_{i}$ 's are part of a system of regular parameters at the strict localization of $X$ at $\bar{x}$. In [56], Rapoport and Zink proved that the absolute purity assumption needed to justify the calculation (2.5) was satisfied, and, moreover, that the action of the inertia $I$ on the nearby cycles groups $R^{q} \Psi \Lambda$ was tame, hence trivial (by (2.5)). It follows that $I$ acts through its tame quotient $I_{\mathrm{t}}$, and if $T$ is a topological generator of $I_{\mathrm{t}}$, the action of $T-1$ on $R \Psi \Lambda$ is nilpotent. In fact, imitating a construction of Steenbrink, they gave an explicit description of this action, using a realization of $R \Psi \Lambda$ as the total complex of a certain bicomplex (the Rapoport-Zink bicomplex), at least in the strict semistable reduction case, i.e., when the special fiber $X_{s}$ is a sum of smooth components $D_{i}, 1 \leqslant i \leqslant m$ (see [34], [56]).

If $d=\operatorname{dim}\left(X_{s}\right)=\operatorname{dim}\left(X_{\eta}\right)$ is the relative dimension of $X / S$, one has $(T-1)^{d+1}=0$ on $R \Psi \Lambda$. As $\Lambda_{X_{\eta}}[d]$ is perverse, so is $R \Psi \Lambda[d]$ by $(6.2)$. When $d \leqslant 1$, or $m \leqslant 2$ (in which cases $(T-1)^{2}=0$ ), or $\Lambda=\mathbf{Q}_{\ell}$, the monodromy operator

$$
N: R \Psi \Lambda(1) \longrightarrow R \Psi \Lambda
$$

such that $\sigma=\exp \left(N t_{\ell}(\sigma)\right)$ for all $\sigma \in I$ is defined, and it is more convenient to work with $N$, which does not depend of the choice of a generator of $I_{t}$, and is Galois equivariant. Let $\operatorname{Per}\left(X_{\bar{s}}\right)$ denote the category of perverse sheaves on $X_{\bar{s}}$. As $N$ is a (twisted) nilpotent endomorphism of $R \Psi \Lambda$ in the abelian category $\operatorname{Per}\left(X_{\bar{s}}\right)[-d]$, it defines a monodromy filtration

$$
\cdots \subset M_{i} \subset M_{i+1} \subset \ldots
$$


characterized by $N M_{i}(1) \subset M_{i-2}$, and $N^{i}: \operatorname{gr}_{i}^{M} \stackrel{\sim}{\rightarrow} \operatorname{gr}_{-i}^{M}(-i)$ for $i \geqslant 0$. As a by-product of [56], T. Saito [62] calculated the associated graded object:

$$
\operatorname{gr}_{k}^{M} R \Psi \Lambda=\bigoplus_{p-q=k, p \geqslant 0, q \geqslant 0} K_{p, q}
$$

with

$$
K_{p, q}:=\left(a_{p+q *} \Lambda\right)[-p-q](-p)
$$

where

$$
a_{n}: X_{s}^{(n)}:=\coprod_{J \subset\{1, \ldots, m\},|J|=n+1} \bigcap_{i \in J} D_{i} \rightarrow X_{s}
$$

is the natural projection. The operator $N: \operatorname{gr}_{k}^{M} \rightarrow \operatorname{gr}_{k-2}^{M}(-1)$ sends $K_{p, q}$ to $K_{p-1, q+1}(-1)$ by the identity ${ }^{(20)}$.

Example. - If $m=2$, so that $X_{s}$ consists of a pair $D_{1}, D_{2}$ of smooth

\begin{tabular}{|c|c|c|}
\hline $\operatorname{gr}_{1}$ & & $\Lambda_{C}[-1](-1)$ \\
\hline $\mathrm{gr}_{0}$ & $\Lambda_{D_{1}} \oplus \Lambda_{D_{2}}$ & $N$ \\
\hline $\mathrm{gr}_{-1}$ & & $\begin{array}{c}\downarrow \\
\Lambda_{C}[-1]\end{array}$ \\
\hline
\end{tabular}
divisors (of dimension $d$ ) crossing transversally, we have the following picture, where $C=D_{1} \cap D_{2}$ :

where the isomorphism $N: \operatorname{gr}_{1} \stackrel{\sim}{\rightarrow} \operatorname{gr}_{-1}(-1)$ is the identity of $\Lambda_{C}[-1](-1)$. Note that $\Lambda_{D_{1}} \oplus \Lambda_{D_{2}}=\underline{I C} \underline{X}_{s}[-d]$, where $\underline{I C}_{X_{s}}$ is the intersection complex of $X_{s}$, i.e., $j_{! *}(\Lambda[d])\left(j: X_{s}-C \hookrightarrow X_{s}\right.$ the inclusion). The object (6.4) appears in the Picard-Lefschetz formula in odd relative dimension (cf. [36] ${ }^{(21)}$ ). The simplest case is $X=S\left[t_{1}, t_{2}\right] /\left(t_{1} t_{2}-\pi\right)$. It is sometimes called the PicardLefschetz oscillator (cf. [65]), as the triple $\left(\mathrm{gr}_{1}, \mathrm{gr}_{-1}, N\right)$ uniquely extends to the standard representation of $\mathrm{SL}_{2}$ over $\Lambda$.

6.4. The weight-monodromy conjecture. Let $X / S$ be proper, with strict semistable reduction. The monodromy filtration (6.2) induces a spectral sequence, called the weight spectral sequence

$$
E_{1}^{i, j}=H^{i+j}\left(X_{\bar{s}}, \operatorname{gr}_{-i}^{M} R \Psi \Lambda\right) \Longrightarrow H^{i+j}\left(X_{\bar{\eta}}\right),
$$

(20) See also [37] for an alternate proof of the tameness of $R \Psi \Lambda$ and an exposition of the above calculations.

(21) There is a typo on p. 251, 1. 18: $|i|>-1$ should be replaced by $|i|>1$. 
whose initial term can be re-written

$$
E_{1}^{-r, n+r}=\bigoplus_{q \geqslant 0, r+q \geqslant 0} H^{n-r-2 q}\left(X_{\bar{s}}^{(r+1+2 q)}\right)(-r-q)
$$

thanks to (6.3). The differential $d_{1}$ is a sum of restriction and Gysin maps. Note that though $\left(E_{1}, d_{1}\right)$ depends only on $X_{s},(6.5)$ does depend on $X^{(22)}$.

The weight spectral sequence degenerates at $E_{2}$. This was first proved for $k$ finite [56], as a consequence of Weil $\mathrm{II}^{(23)}$. The general case was proved by Nakayama [54] and Ito [43], independently.

Let $\widetilde{M}_{\bullet}$ be the abutment filtration of (6.5). For $m \in \mathbf{Z}$, the monodromy operator $N$ on $H^{m}\left(X_{\bar{\eta}}\right)$ sends $\widetilde{M}_{i}$ to $\widetilde{M}_{i-2}(-1)$. A central problem in the theory is the following conjecture, called the weight monodromy conjecture:

Conjecture 6.1. - Assume that $\Lambda=\mathbf{Q}_{\ell}$. Then, for all $m \in \mathbf{Z}$, the filtration $\widetilde{M}_{\bullet}$ on $H^{m}\left(X_{\bar{\eta}}\right)$ is the monodromy filtration $M_{\bullet}$ associated with the nilpotent operator $N$, i.e., for all $i \geqslant 0, N^{i}: \operatorname{gr}_{i}^{\widetilde{M}} H^{m} \stackrel{\sim}{\rightarrow} \operatorname{gr}_{-i}^{\widetilde{M}} H^{m}(-i)$.

By the description of $N$ given in (6.3), $N$ induces isomorphisms at the $E_{1}$ level. As (6.5) degenerates at $E_{2}, 6.1$ is equivalent to saying that $N$ induces isomorphisms at the $E_{2}$-level. When $k$ is finite, it follows from Weil II that $\widetilde{M}_{\bullet}$ on $H^{n}\left(X_{\bar{\eta}}\right)$ is the weight filtration, up to shift: $\widetilde{M}_{r}$ is the piece of weight $\leqslant r+n$. Therefore, in this case, Conjecture 6.1 is equivalent to saying that the monodromy filtration $M_{\bullet}$ is pure, i.e., the graded pieces of $_{\mathrm{gr}_{\bullet}^{M}}$ are pure.

Here is the status of Conjecture 6.1:

- for $k$ finite, $X / S$ coming by localization from a proper, flat scheme over a smooth curve over $k$, with semistable reduction at a closed point, Conjecture 6.1 was proved by Deligne ([21, 1.8.5]);

- in the general equicharacteristic $p$ case, by Ito [43];

- for $k$ finite and $\operatorname{dim}(X / S) \leqslant 1$ (resp. $\operatorname{dim}(X / S) \leqslant 2$ ) by Grothendieck (Corollary 4.3(b)) (resp. by Rapoport-Zink ([56, 2.13, 2.23]);

- for certain 3 -folds $X_{\eta}$, and certain $p$-adically uniformized varieties $X_{\eta}([41],[42])$;

- for $X_{\eta}$ a set-theoretic complete intersection in a projective space (or in a smooth projective toric variety), by Scholze [66].

The general case is still open.

(22) actually only via $X \otimes R /\left(\pi^{2}\right)$, where $S=\operatorname{Spec} R$, as shown by Nakayama [53].

(23) The complex analogue had been proved by Steenbrink [69], using Hodge theory. 
6.5. Euler-Poincaré characteristics of $\ell$-adic sheaves. Deep relations between $\ell$-adic nearby cycles and global Euler-Poincaré characteristics were discovered by Deligne in the mid 1970's, spurring a new line of research which has been active for the past forty years. See ([38], [40]) for (partial) surveys. Breakthroughs were made recently by Beilinson [5] and T. Saito [63] in their work on singular supports and characteristic cycles of $\ell$-adic sheaves.

6.6. Vanishing cycles over higher dimensional bases. Though, in general, vanishing cycles don't behave well in families, in the early 1980's Deligne proposed a theory of functors $R \Psi$ and $R \Phi$ over general bases. It was summarized in [46], and revisited and completed in [39], [55]. This formalism is used in [27] and [63].

\section{Bounds for the exponent of unipotence (after Gabber)}

This section is devoted to the proof of Theorem 2.3. This proof is due to Gabber.

The following lemma is well known. We give it for lack of a suitable reference.

LEMMA 7.1. - Let $S=(S, s, \eta)$ be a strictly local trait, $\Lambda$ be as in 1.1, $(X, Z)$ a strict semistable pair over $S$ in the sense of de Jong $([45,6.3]$. Let $u: X-Z_{f} \hookrightarrow X$ be the open immersion, where $Z_{f}$ is the horizontal part of $Z$, in de Jong's notation. Then, for all $q \in \mathbf{Z}$, the inertia group $I$ acts trivially on $R^{q} \Psi_{X}\left(R u_{\eta *} \Lambda\right)$ (resp. $\left.R^{q} \Psi_{X}\left(u_{\eta !} \Lambda\right)\right)$.

Proof. - The assertion relative to $R u_{\eta *} \Lambda$ is a particular case of $[53,3.5]$ (see also $[35,8.4 .4])$. A direct proof can be given as follows. The conclusion has to be checked on the stalks at geometric points $x$ of $X_{s}$. By the local description of strict semistable pairs $([45,6.4])$, étale locally at such a point $x, X$ is isomorphic to $X_{1} \times_{S} X_{2}$, where $X_{1}=S\left[t_{1}, \ldots, t_{n}\right] /\left(t_{1} \ldots t_{n}-\pi\right)$, $X_{2}=S\left[s_{1}, \ldots, s_{m}\right]$, and the horizontal part $Z_{f}$ is $X_{1} \times_{S} D$, where $D=$ $S\left[s_{1}, \ldots, s_{m}\right] /\left(s_{1} \ldots s_{r}\right)$, with $1 \leqslant r \leqslant m$, and $\pi$ is a uniformizing parameter of $S$. We may therefore assume that $X=X_{1} \times{ }_{S} X_{2}$ and $Z_{f}=X_{1} \times{ }_{S} D$. Then $X_{\eta}-Z_{\eta}=X_{1} \times_{S}\left(X_{2}-D\right)$, and $u=\operatorname{Id}_{X_{1}} \times_{S} v$, where $v: X_{2}-D \hookrightarrow X_{2}$ is the inclusion. As $R u_{\eta *} \Lambda=\Lambda_{X_{1 \eta}} \otimes R v_{\eta *} \Lambda$ (smooth base change), the commutation of $R \Psi$ with external tensor products ([34, 4.7]) implies:

$$
R \Psi_{X}\left(R u_{\eta *} \Lambda\right)=R \Psi_{X_{1}}(\Lambda) \otimes^{L} R \Psi_{X_{2}}\left(R v_{\eta *} \Lambda\right) .
$$


As $X_{2}$ is smooth over $S$ and $D$ is a relative divisor with normal crossings in $X_{2}, R \Phi_{X_{2}}\left(R v_{\eta *} \Lambda\right)=0^{(24)}$, so that

$$
R \Psi_{X_{2}}\left(R v_{\eta *} \Lambda\right)=R v_{s *} \Lambda,
$$

and in particular $I$ acts trivially on $R \Psi_{X_{2}}\left(R v_{\eta *} \Lambda\right)$. On the other hand, $I$ acts trivially on $R^{q} \Psi_{X_{1}}(\Lambda)$ for all $q$ (cf. 6.3) for all $q$. Moreover, by (2.5), the stalks of $R^{q} \Psi_{X_{1}}(\Lambda)$ are finitely generated and free over $\Lambda$, and the same is true of the $R^{q} \Psi_{X_{2}}\left(R v_{\eta *} \Lambda\right)$ by (7.2). Therefore, by Künneth, (7.1) gives

$$
R^{q} \Psi_{X}\left(R u_{\eta *} \Lambda\right)=\bigoplus_{i+j=q} R^{i} \Psi_{X_{1}}(\Lambda) \otimes R^{j} v_{s *}(\Lambda) .
$$

As $I$ acts trivially on both factors of each summand in the right hand side, the conclusion follows in this case. Similarly, we have

$$
R \Psi_{X}\left(u_{\eta !} \Lambda\right)=R \Psi_{X_{1}}(\Lambda) \otimes^{L} R \Psi_{X_{2}}\left(v_{\eta !} \Lambda\right),
$$

and

$$
R \Psi_{X_{2}}\left(v_{\eta !} \Lambda\right)=v_{s !} \Lambda,
$$

$\left([22 \text {, vol. II, XIII 2.1.11, p. 105]) })^{(25)}\right.$. So (7.4) and (7.5) yield

$$
R \Psi_{X}\left(u_{\eta !} \Lambda\right)=u_{s !} R \Psi_{X-Z_{f}}(\Lambda) .
$$

In particular, $I$ acts trivially on $R^{q} \Psi_{X}\left(u_{\eta !} \Lambda\right)$ for all $q$, which finishes the proof.

The next lemma is also more or less standard, but again we couldn't find a suitable reference ${ }^{(26)}$. Its statement and proof are due to Gabber.

Lemma 7.2. - Let $S=(S, s, \eta)$ be a strictly local complete trait. Let $\bar{X}$ be a proper scheme over $S$, which is a compactification of an open subscheme $X_{\eta}$ of its generic fiber $\bar{X}_{\eta}$. Let $n \in \mathbf{N}$. Then there exists a finite extension of traits $S^{\prime}=\left(S^{\prime}, s^{\prime}, \eta^{\prime}\right) \rightarrow S$, a proper simplicial scheme $\bar{X}^{\prime}$. over $S^{\prime}$, an $S^{\prime}$-map $h_{\bullet}: \bar{X}_{\bullet}^{\prime} \rightarrow \bar{X}^{\prime}:=\bar{X} \times_{S} S^{\prime}$ satisfying the following conditions:

(i) The morphism $\bar{X}_{\bullet}^{\prime} \rightarrow \bar{X}^{\prime}$ induced by $h_{\bullet}$ is a proper hypercovering;

(ii) For $0 \leqslant r \leqslant n$, if $C$ is a connected component of $\bar{X}_{r}^{\prime}$, then either $C \times{ }_{\bar{X}^{\prime}} X_{\eta^{\prime}}^{\prime}=\emptyset\left(\right.$ where $\left.X_{\eta^{\prime}}^{\prime}:=X_{\eta} \times{ }_{\eta} \eta^{\prime}\right)$, or there exists a strictly local complete trait $S^{\prime \prime}$ between $S$ and $S^{\prime}$, a strict semistable pair $(Y, Z)$ over $S^{\prime \prime}$ such that $C=Y \times_{S^{\prime \prime}} S^{\prime}$ and $C \times_{\bar{X}^{\prime}} X_{\eta^{\prime}}^{\prime}=(Y-Z) \times_{S^{\prime \prime}} S^{\prime}$.

(24) As can be checked by induction on $r$, using relative purity, see ([64, Prop. 3.15]) for a generalization.

(25) It can also be deduced from (7.2) by duality, as $R \Psi$ commutes with duality. See again ([64, Prop. 3.15]) for a generalization.

(26) The closest one seems to be $([11,2.2])$, but it doesn't suffice, as the authors assume $X_{\eta}=\bar{X}_{\eta}$. 
Proof. - We will prove, by induction on $n$, the existence of $S^{\prime}$ and $h$ • satisfying (i), (ii), and in addition,

(iii) The $n$-truncated simplicial scheme $\bar{X}_{\leqslant n}^{\prime}$ is split, in the sense of $[2$, Vbis, 5.1.1].

Assume first $n=0$. Decompose the set $I$ of reduced irreducible components $C_{i}(1 \leqslant i \leqslant r)$ of $\bar{X}$ into $I=I_{1} \amalg I_{2}$, where, for $i \in I_{1}, C_{i} \cap X_{\eta}=\emptyset$, and for $i \in I_{2}, C_{i} \cap X_{\eta} \neq \emptyset$. For each $i \in I_{2}$, apply $([45,6.5])$ to the pair consisting of $C_{i}$ and the (proper) closed subset $C_{i}-\left(C_{i} \cap X_{\eta}\right)$. We find a finite extension of traits $S_{i}^{\prime \prime} \rightarrow S$, a strict semistable pair $\left(C_{i}^{\prime \prime}, Z_{i}\right)$ over $S_{i}^{\prime \prime}$, with geometrically irreducible generic fiber $C_{i \eta_{i}^{\prime \prime}}^{\prime \prime}$, an alteration $C_{i}^{\prime \prime} \rightarrow C_{i}$ over $S$, such that $C_{i}^{\prime \prime} \times_{\bar{X}_{i}^{\prime \prime}} X_{i \eta_{i}^{\prime \prime}}^{\prime \prime}=C_{i}^{\prime \prime}-Z_{i}$. As the generic fiber of $C_{i}^{\prime \prime}$ remains connected after any finite extension of traits, we can find a common finite extension $S_{1}$ of the $S_{i}^{\prime \prime}$ such that the components $C_{i}^{\prime \prime} \times_{S_{i}^{\prime \prime}} S_{1}$ satisfy condition (ii). However, $C_{i}^{\prime \prime} \times_{S_{i}^{\prime \prime}} S_{1} \rightarrow C_{i} \times_{S} S_{1}$ is not necessarily surjective. To correct this, we proceed as in the proof of $([11,2.2])$. We take a finite extension $S^{\prime}$ of $S_{1}$, normal over $S$ with group $G:=\operatorname{Aut}\left(S^{\prime} / S\right)$ and replace $C_{i}^{\prime \prime} \times{ }_{S_{i}^{\prime \prime}} S_{1}$ by the disjoint sum, for $g \in G$, of the $C_{i, g}^{\prime \prime}$ deduced from $C_{i}^{\prime \prime} \times{ }_{S_{i}^{\prime \prime}} S_{1}$ by base change by the composite $S^{\prime} \stackrel{g}{\rightarrow} S^{\prime} \rightarrow S_{1}$. Denote this disjoint sum by $C_{i}^{\prime}$. Let

$$
\bar{X}_{0}^{\prime}:=\left(\coprod_{i \in I_{1}} C_{i} \times{ }_{S} S^{\prime}\right) \coprod\left(\coprod_{i \in I_{2}} C_{i}^{\prime}\right)
$$

Then the map

$$
\operatorname{cosk}_{0}\left(\bar{X}_{0}^{\prime} / \bar{X}^{\prime}\right) \longrightarrow \bar{X}^{\prime}
$$

satisfies conditions (i) and (ii) (and trivially (iii)) for $n=0$.

Assume now that Lemma 7.2 has been proved up to $n$, and let us prove it for $n+1$. Take $S^{\prime}, h_{\bullet}: \bar{X}_{\bullet}^{\prime} \rightarrow \bar{X}^{\prime}:=\bar{X} \times_{S} S^{\prime}$ satisfying conditions (i), (ii), (iii). Let us construct a finite extension $T$ of $S^{\prime}$, with generic point $\zeta$, and $v_{\bullet}: V_{\bullet} \rightarrow \bar{X} \times_{S} T$ satisfying conditions (i), (ii), (iii) up to $n+1$ for $S^{\prime}$ replaced by $T$ and $\left(\bar{X}^{\prime}, X_{\eta^{\prime}}^{\prime}\right)$ replaced by $\left(\bar{X} \times{ }_{S} T, X_{\eta} \times{ }_{\eta} \zeta\right)$. Note that these conditions are stable under finite extensions of traits. Let

$$
W:=\left(\operatorname{cosk}_{n}\left(\bar{X}_{\leqslant n}^{\prime} / \bar{X}^{\prime}\right)\right)_{n+1}
$$

We proceed as before with $\left(W, W \times_{\bar{X}^{\prime}} X_{\eta^{\prime}}^{\prime}\right)$ in place of $\left(\bar{X}, X_{\eta}\right)$. We find a finite extension $T$ of $S^{\prime}$, a proper surjective morphism $W_{n+1} \rightarrow W_{T}:=$ $W \times{ }_{S^{\prime}} T$, for which the connected components of $W_{n+1}$ satisfy (ii) (relative to $T)$. Then, as in the proof of $[11,2.2]$, we extend the split $n$-truncated simplicial scheme $V_{\leqslant n}:=\bar{X}_{\leqslant n}^{\prime} \times_{S^{\prime}} T$ over $\bar{X}_{T}^{\prime}:=\bar{X} \times_{S} T$ to a split $(n+1)$ truncated simplicial scheme $V_{\leqslant n+1}$ over $\bar{X}_{T}^{\prime}$ by [2, Vbis, 5.1.3], namely, by 
putting

$$
V_{n+1}:=W_{n+1} \coprod\left(\coprod_{[n+1] \rightarrow[i], i \leqslant n} N\left(V_{i}\right)\right)
$$

where $N\left(V_{i}\right)$ is the complement of the union of the images of the degeneracy morphisms with target $V_{i}$, and defining face and degeneracy operators between $V_{n+1}$ and $V_{n}$ as in [2]. Finally, we define

$$
V_{\bullet}:=\operatorname{cosk}_{n+1}\left(V_{\leqslant n+1} / \bar{X}_{T}^{\prime}\right) \text {, }
$$

and $v_{\bullet}: V_{\bullet} \rightarrow \bar{X} \times_{S} T$ to be the canonical extension of $V_{\leqslant n+1} \rightarrow \bar{X}_{T}^{\prime}=$ $\bar{X} \times_{S} T$. The pair $\left(V_{\bullet}, v_{\bullet}\right)$ over $T$ satisfies conditions (i), (ii), (iii) up to $n+1$.

7.1. Let's prove Theorem 2.3. We may assume $S$ strictly local, and, furthermore, complete (which doesn't change the inertia $I$ nor $H^{*}\left(X_{\bar{\eta}}, \Lambda\right.$ ) (resp. $\left.H_{c}^{*}\left(X_{\bar{\eta}}, \Lambda\right)\right)$. We may assume $X_{\eta}$ is nonempty. Let $d$ be its dimension. Recall that

$$
H^{i}\left(X_{\bar{\eta}}, \Lambda\right)=H_{c}^{i}\left(X_{\bar{\eta}}, \Lambda\right)=0
$$

for $i>2 d([2, \mathrm{X} 4.3])$. Apply Lemma 7.2 for an integer $n \geqslant 2 d$ and a compactification $\bar{X}$ of $X_{\eta}$ over $S$. Let $u: X_{\eta} \hookrightarrow \bar{X}_{\eta}$ be the open immersion. Take a finite extension $S^{\prime}$ of $S$, a proper simplicial scheme $\bar{X}_{\bullet}^{\prime}$ over $S^{\prime}$, and an $S^{\prime}$-map $h_{\bullet}: \bar{X}_{\bullet}^{\prime} \rightarrow \bar{X}^{\prime}=\bar{X} \times{ }_{S} S^{\prime}$ satisfying conditions (i) and (ii) for $n$. Consider the cartesian square

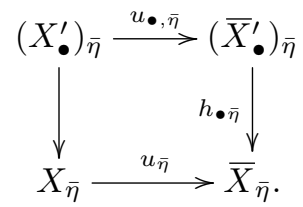

Let $I_{1}=\operatorname{Gal}\left(\bar{\eta} / \eta^{\prime}\right)$. It suffices to show that for any $0 \leqslant m \leqslant 2 d$, and $g \in I_{1}$, $(g-1)^{m+1}=0$ on $H^{m}\left(X_{\bar{\eta}}, \Lambda\right)$ and $H_{c}^{m}\left(X_{\bar{\eta}}, \Lambda\right)$. We have

$$
\begin{gathered}
H^{m}\left(X_{\bar{\eta}}, \Lambda\right)=H^{m}\left(\bar{X}_{\bar{\eta}}, R u_{*} \Lambda\right), \\
H_{c}^{m}\left(X_{\bar{\eta}}, \Lambda\right)=H^{m}\left(\bar{X}_{\bar{\eta}}, u_{!} \Lambda\right) .
\end{gathered}
$$

As $h_{\bullet}$ is a proper hypercovering of $\bar{X}^{\prime}$, hence $h_{\bullet} \bar{\eta}$ a proper hypercovering of $\bar{X}_{\bar{\eta}}=\bar{X}_{\bar{\eta}}^{\prime}$, by cohomological descent and proper base change, we deduce from $(7.7)$

$$
\begin{aligned}
H^{m}\left(\bar{X}_{\bar{\eta}}, R u_{*} \Lambda\right) & =H^{m}\left(\bar{X}_{\bullet \bar{\eta}}^{\prime}, R\left(u_{\bullet}\right)_{*} \Lambda\right), \\
H^{m}\left(\bar{X}_{\bar{\eta}}, u_{!} \Lambda\right) & =H^{m}\left(\bar{X}_{\bullet \bar{\eta}}^{\prime},\left(u_{\bullet \bar{\eta}}\right) ! \Lambda\right) .
\end{aligned}
$$

As $\bar{X}_{\bullet}^{\prime}$ is proper over $S^{\prime}$, we have

$$
H^{m}\left(\bar{X}_{\bullet \bar{\eta}}^{\prime}, R\left(u_{\bullet \bar{\eta}}\right)_{*} \Lambda\right)=H^{m}\left(\bar{X}_{\bullet s^{\prime}}^{\prime}, R \Psi R\left(u_{\bullet \bar{\eta}}\right)_{*} \Lambda\right),
$$


where $R \Psi$ is relative to $\bar{X}_{\bullet}^{\prime}$ over $S^{\prime}$ (we use here a (straightforward) extension of the formalism of $R \Psi$ to simplicial schemes). Similarly,

$$
H^{m}\left(\bar{X}_{\bullet \bar{\eta}}^{\prime},\left(u_{\bullet \bar{\eta}}\right) ! \Lambda\right)=H^{m}\left(\bar{X}_{\bullet \bar{s}^{\prime}}^{\prime}, R \Psi\left(u_{\bullet \bar{\eta}}\right) ! \Lambda\right) .
$$

Therefore, by the spectral sequences

$$
\begin{gathered}
E_{2}^{i j}=H^{i}\left(\bar{X}_{\bullet \bar{s}^{\prime}}^{\prime}, R^{j} \Psi R\left(u_{\bullet \bar{\eta}}\right)_{*} \Lambda\right) \Longrightarrow H^{i+j}\left(\bar{X}_{\bullet \bar{s}^{\prime}}^{\prime}, R \Psi R\left(u_{\bullet \bar{\eta}}\right)_{*} \Lambda\right), \\
E_{2}^{i j}=H^{i}\left(\bar{X}_{\bullet \bar{s}^{\prime}}^{\prime}, R^{j} \Psi\left(u_{\bullet \bar{\eta}}\right)_{!} \Lambda\right) \Longrightarrow H^{i+j}\left(\bar{X}_{\bullet \bar{s}^{\prime}}^{\prime}, R \Psi\left(u_{\bullet \bar{\eta}}\right)_{!} \Lambda\right),
\end{gathered}
$$

it suffices to show that for all $g \in I_{1}, g$ acts trivially on $E_{2}^{i j}$ for $0 \leqslant$ $i, j \leqslant m$ and $i+j=m$. The map defined by $g-1$ on $E_{2}^{i j}$ factors through $H^{i}\left(\bar{X}_{\bullet}^{\prime} \bar{s}^{\prime},(g-1) R^{j} \Psi R\left(u_{\bullet}\right)_{*} \Lambda\right)\left(\operatorname{resp} . H^{i}\left(\bar{X}_{\bullet}^{\prime} \bar{\bullet}^{\prime},(g-1) R^{j} \Psi\left(u_{\bullet}\right)_{!} \Lambda\right)\right)$. Therefore it suffices to show

$$
H^{i}\left(\bar{X}_{\bullet}^{\prime} \bar{s}^{\prime},(g-1) R^{j} \Psi R\left(u_{\bullet}\right)_{*} \Lambda\right)=H^{i}\left(\bar{X}_{\bullet}^{\prime} \bar{s}^{\prime},(g-1) R^{j} \Psi\left(u_{\bullet}\right)_{!} \Lambda\right)=0
$$

for $0 \leqslant i, j \leqslant m, i+j=m$. Now, for any sheaf of $\Lambda$-modules $\mathcal{F}_{\bullet}$ on $\bar{X}_{\bullet \bar{s}^{\prime}}^{\prime}$, we have the descent spectral sequence

$$
E_{1}^{a b}=H^{b}\left(\bar{X}_{a, \bar{s}^{\prime}}^{\prime}, \mathcal{F}_{a}\right) \Longrightarrow H^{a+b}\left(\bar{X}_{\bullet}^{\prime} \bar{s}^{\prime}, \mathcal{F}_{\bullet}\right) .
$$

In particular, given $i \geqslant 0$, if for all $0 \leqslant a \leqslant i, \mathcal{F}_{a}=0$, then $H^{i}\left(\bar{X}_{\bullet}^{\prime} \bar{s}^{\prime}, \mathcal{F}_{\bullet}\right)=0$. Therefore, to show $(*)$ we need only to prove that, for $0 \leqslant i \leqslant m$ (and any $j$ ), we have

$$
(g-1) R^{j} \Psi R\left(u_{i \bar{\eta}}\right)_{*} \Lambda=(g-1) R^{j} \Psi\left(u_{i \bar{\eta}}\right) ! \Lambda=0
$$

(on $\bar{X}_{i \bar{s}^{\prime}}^{\prime}$ ). This is checked at geometric points $\bar{x}$ of $\bar{X}_{i \bar{s}^{\prime}}^{\prime}$. As $i \leqslant m \leqslant 2 d \leqslant n$, $\bar{X}_{i}^{\prime}$ satisfies condition (ii) of Lemma 7.2. If $\bar{x}$ is above a point $x$ of a connected component $C$ such that $C \times_{\bar{X}^{\prime}} X_{\eta^{\prime}}^{\prime}=\emptyset$ (hence $C \times_{\bar{X}^{\prime}} \bar{X}_{\eta^{\prime}}^{\prime}=\emptyset$ ), then

$$
R \Psi\left(R u_{i, *} \Lambda\right)_{\bar{x}}=R \Psi\left(u_{i, !} \Lambda\right)_{\bar{x}}=0,
$$

and there is nothing to prove. Otherwise, $\bar{x}$ is above a point $x$ of a component $C$ satisfying the conditions stated in (ii) relative to a semistable pair $(Y, Z)$ over $S^{\prime \prime}$. Then, if $I^{\prime \prime}:=\operatorname{Gal}\left(\bar{\eta} / \eta^{\prime \prime}\right)$ (a group containing $I_{1}$ ), by Lemma 7.1 $I^{\prime \prime}$ acts trivially on $R^{j} \Psi\left(R u_{i, *} \Lambda\right)_{\bar{x}}$ and $R^{j} \Psi\left(u_{i, !} \Lambda\right)_{\bar{x}}$, hence $(* *)$ is satisfied at $\bar{x}$. This completes the proof of Theorem 2.3 .

\section{Bibliography}

[1] V. I. ARNol'D, "Critical points of smooth functions", in Proceedings of the International Congress of Mathematicians (Vancouver, B. C., 1974), Vol. 1, 1975, p. 19-39.

[2] M. Artin, A. Grothendieck \& J.-L. Verdier, Théorie des topos et cohomologie étale des schémas, Lecture Notes in Mathematics, vol. 269, 270, 305, Springer, 1972, 1973, Séminaire de Géométrie Algébrique du Bois-Marie 1963-1964 (SGA 4), avec la collaboration de N. Bourbaki, P. Deligne et B. Saint-Donat, xix +525 pages.

[3] M. Artin \& G. Winters, "Degenerate fibres and stable reduction of curves", Topology 10 (1971), p. 373-383. 
[4] L. BÉGueRI, Dualité sur un corps local à corps résiduel algébriquement clos, Mém. Soc. Math. Fr., Nouv. Sér., vol. 4, Gauthier-Villars, 1980, 121 pages.

[5] A. Beilinson, "Constructible sheaves are holonomic", Sel. Math., New Ser. 22 (2016), no. 4, p. 1797-1819.

[6] A. Beilinson \& J. Bernstein, "A proof of Jantzen conjectures", in I. M. Gelfand Seminar, Advances in Soviet Mathematics, vol. 16, American Mathematical Society, 1993, p. 1-50.

[7] A. Beilinson, J. Bernstein, P. Deligne \& O. Gabber, Faisceaux pervers, 2nd ed., Astérisque, vol. 100, Société Mathématique de France, 2018, vi+180 pages.

[8] A. Bertapelle, "On perfectness of Grothendieck's pairing for the $l$-parts of component groups", J. Reine Angew. Math. 538 (2001), p. 223-236.

[9] A. Bertapelle \& S. Bosch, "Weil restriction and Grothendieck's duality conjecture", J. Algebr. Geom. 9 (2000), no. 1, p. 155-164.

[10] P. Berthelot, "Altérations de variétés algébriques (d'après A. J. de Jong)", in Séminaire Bourbaki, Vol. 1995/96, Astérisque, vol. 241, Société Mathématique de France, 1997, Exp. No. 815, p. 273-311.

[11] P. Berthelot, H. Esnault \& K. Rülling, "Rational points over finite fields for regular models of algebraic varieties of Hodge type $\geqslant 1 "$, Ann. Math. 176 (2012), no. 1, p. 413-508.

[12] S. Bosch, "Component groups of abelian varieties and Grothendieck's duality conjecture", Ann. Inst. Fourier 47 (1997), no. 5, p. 1257-1287.

[13] S. Bosch \& D. Lorenzini, "Grothendieck's pairing on component groups of Jacobians", Invent. Math. 148 (2002), no. 2, p. 353-396.

[14] S. Bosch, W. Lütкebohmert \& M. Raynaud, Néron models, Ergebnisse der Mathematik und ihrer Grenzgebiete. 3. Folge., vol. 21, Springer, 1990, x+325 pages.

[15] P. Colmez \& J.-P. Serre (eds.), Correspondance Grothendieck-Serre, Documents Mathématiques, vol. 2, Société Mathématique de France, 2001, xii+288 pages.

[16] P. Deligne, "Théorie de Hodge. I", in Actes du Congrès International des Mathématiciens (Nice, 1970), Tome 1, Gauthier-Villars, 1971, p. 425-430.

[17] — "Théorie de Hodge. II", Publ. Math., Inst. Hautes Étud. Sci. (1971), no. 40, p. $5-57$.

[18] — "La conjecture de Weil. I", Publ. Math., Inst. Hautes Étud. Sci. (1974), no. 43 , p. $273-307$.

[19] — "Théorie de Hodge. III", Publ. Math., Inst. Hautes Étud. Sci. (1974), no. 44, p. $5-77$.

[20] - Cohomologie étale, Lecture Notes in Mathematics, vol. 569, Springer, 1977, Séminaire de Géométrie Algébrique du Bois-Marie SGA $4 \frac{1}{2}$, iv +312 pages.

[21] — "La conjecture de Weil. II", Publ. Math., Inst. Hautes Étud. Sci. (1980), no. 52 , p. $137-252$.

[22] P. Deligne, A. Grothendieck \& N. Katz, Groupes de monodromie en géométrie algébrique, Lecture Notes in Mathematics, vol. 288, 340, Springer, 1972, 1973, Séminaire de Géométrie Algébrique du Bois-Marie 1967-1969 (SGA 7), (SGA 7 I) dirigé par A. Grothendieck, avec la collaboration de M. Raynaud et D. S. Rim, ix+523 pages; (SGA 7 II), dirigé par P. Deligne et N. Katz, $x+438$ pages.

[23] P. Deligne \& D. Mumford, "The irreducibility of the space of curves of given genus", Publ. Math., Inst. Hautes Étud. Sci. (1969), no. 36, p. 75-109.

[24] M. Demazure, J. Giraud \& M. Raynaud, "Schémas abéliens", Séminaire Orsay. Séminaire de géométrie algébrique 1967-1968. Faculté des Sciences de l'université de Paris-Orsay. Available from the portal of Biblothèque Jacques Hadamard, département de mathématiques d'Orsay: https://bibliotheque.math.u-psud.fr. 
[25] L. Fu, Etale cohomology theory, revised ed., Nankai Tracts in Mathematics, vol. 14, World Scientific, 2015, $\mathrm{x}+611$ pages.

[26] K. Fujiwara, "A proof of the absolute purity conjecture (after Gabber)", in Algebraic geometry 2000, Azumino (Hotaka), Advanced Studies in Pure Mathematics, vol. 36, Mathematical Society of Japan, 2002, p. 153-183.

[27] A. Genestier \& V. Lafforgue, "Chtoucas restreints pour les groupes réductifs et paramétrisation de Langlands locale", https://arxiv.org/abs/1709.00978, 2018.

[28] P. A. Griffiths, "Periods of integrals on algebraic manifolds: Summary of main results and discussion of open problems", Bull. Am. Math. Soc. 76 (1970), p. 228296.

[29] - "A transcendental method in algebraic geometry", in Actes du Congrès International des Mathématiciens (Nice, 1970), Tome 1, Gauthier-Villars, 1971, p. 113119 .

[30] P. A. GRiffiths \& W. Schmid, "Recent developments in Hodge theory: a discussion of techniques and results", in Discrete subgroups of Lie groups and applicatons to moduli (Internat. Colloq., Bombay, 1973), Oxford University Press, 1975, p. 31-127.

[31] A. Grothendieck, Cohomologie l-adique et fonctions L, Lecture Notes in Mathematics, vol. 589, Springer, 1977, Séminaire de Géométrie Algébrique du Bois-Marie 1965-1966 (SGA 5), avec la collaboration de I. Bucur, C. Houzel, L. Illusie, J.-P. Jouanolou et J.-P. Serre, édité par L. Illusie, xii+484 pages.

[32] J.-I. Igusa, "Abstract vanishing cycle theory", Proc. Japan Acad. 34 (1958), p. 589593.

[33] L. Illusie, "Réalisation $l$-adique de l'accouplement de monodromie d'après A. Grothendieck", in Courbes modulaires et courbes de Shimura (Orsay, 1987/1988), Astérisque, vol. 196-197, Société Mathématique de France, 1991, p. 27-44.

[34] — "Autour du théorème de monodromie locale", in Périodes p-adiques (Séminaire de Bures, 1988), Astérisque, vol. 223, Société Mathématique de France, 1994, dirigé par Jean-Marc Fontaine, réédition 2020, p. 9-57.

[35] — "An overview of the work of K. Fujiwara, K. Kato, and C. Nakayama on logarithmic étale cohomology", in Cohomologies p-adiques et applications arithmétiques, II, Astérisque, vol. 279, Société Mathématique de France, 2002, p. 271-322.

[36] - "Sur la formule de Picard-Lefschetz", in Algebraic geometry 2000, Azumino (Hotaka), Advanced Studies in Pure Mathematics, vol. 36, Mathematical Society of Japan, 2002, p. 249-268.

[37] - "On semistable reduction and the calculation of nearby cycles", in Geometric aspects of Dwork theory. Vol. I, II, Walter de Gruyter, 2004, p. 785-803.

[38] , "Miscellany on traces in $\ell$-adic cohomology: a survey", Jpn. J. Math. 1 (2006), no. 1, p. 107-136.

[39] - "Exposé XI. Produits orientés", in Travaux de Gabber sur l'uniformisation locale et la cohomologie étale des schémas quasi-excellents, Astérisque, vol. 363-364, Société Mathématique de France, 2014, p. 213-234.

[40] - "From Pierre Deligne's secret garden: looking back at some of his letters", Jpn. J. Math. 10 (2015), no. 2, p. 237-248.

[41] T. Iто, "Weight-monodromy conjecture for certain threefolds in mixed characteristic", Int. Math. Res. Not. (2004), no. 2, p. 69-87.

[42] , "Weight-monodromy conjecture for $p$-adically uniformized varieties", Invent. Math. 159 (2005), no. 3, p. 607-656.

[43] - "Weight-monodromy conjecture over equal characteristic local fields", Am. J. Math. 127 (2005), no. 3, p. 647-658.

[44] A. J. DE Jong, "Crystalline Dieudonné module theory via formal and rigid geometry", Publ. Math., Inst. Hautes Étud. Sci. 82 (1995), p. 5-96. 
[45] - "Smoothness, semi-stability and alterations", Publ. Math., Inst. Hautes Étud. Sci. (1996), no. 83, p. 51-93.

[46] G. Laumon, "Vanishing cycles over a base of dimension $\geqslant 1$ ", in Algebraic geometry (Tokyo/Kyoto, 1982), Lecture Notes in Mathematics, vol. 1016, Springer, 1983, p. $143-150$.

[47] Q. Lu \& W. Zheng, "Duality and nearby cycles over general bases", Duke Math. J. 168 (2019), no. 16, p. 3135-3213.

[48] Y. I. Manin (ed.), K-theory, arithmetic and geometry, Lecture Notes in Mathematics, vol. 1289, Springer, 1987, Papers from the seminar held at Moscow State University, Moscow, 1984-1986, vi+399 pages.

[49] B. Mazur, "Modular curves and the Eisenstein ideal", Publ. Math., Inst. Hautes Étud. Sci. (1977), no. 47, p. 33-186, With an appendix by B. Mazur and M. Rapoport.

[50] W. G. McCallum, "Duality theorems for Néron models", Duke Math. J. 53 (1986), no. 4, p. 1093-1124.

[51] J. Milnor, Singular points of complex hypersurfaces, Annals of Mathematics Studies, vol. 61, Princeton University Press; University of Tokyo Press, 1968, iii+122 pages.

[52] D. Mumford, "Bi-extensions of formal groups", in Algebraic Geometry (Internat. Colloq., Tata Inst. Fund. Res., Bombay, 1968), Oxford University Press, 1969, p. 307322.

[53] C. Nakayama, "Nearby cycles for log smooth families", Compos. Math. 112 (1998), no. 1 , p. $45-75$.

[54] - "Degeneration of $l$-adic weight spectral sequences", Am. J. Math. 122 (2000), no. 4, p. 721-733.

[55] F. Orgogozo, "Modifications et cycles proches sur une base générale", Int. Math. Res. Not. 2006 (2006), article no. 25315 (38 pages).

[56] M. RAPOPORT \& T. ZINK, "Über die lokale Zetafunktion von Shimuravarietäten. Monodromiefiltration und verschwindende Zyklen in ungleicher Charakteristik", Invent. Math. 68 (1982), no. 1, p. 21-101.

[57] M. Raynaud, Faisceaux amples sur les schémas en groupes et les espaces homogènes, Lecture Notes in Mathematics, vol. 119, Springer, 1970, ii+218 pages.

[58] — "Spécialisation du foncteur de Picard", Publ. Math., Inst. Hautes Étud. Sci. (1970), no. 38, p. 27-76.

[59] — "Variétés abéliennes et géométrie rigide", in Actes du Congrès International des Mathématiciens (Nice, 1970), Tome 1, Gauthier-Villars, 1971, p. 473-477.

[60] K. A. RiBet, "On modular representations of $\mathrm{Gal}(\overline{\mathbf{Q}} / \mathbf{Q})$ arising from modular forms", Invent. Math. 100 (1990), no. 2, p. 431-476.

[61] T. SAito, "Vanishing cycles and geometry of curves over a discrete valuation ring", Am. J. Math. 109 (1987), no. 6, p. 1043-1085.

[62] - "Weight spectral sequences and independence of $l$ ", J. Inst. Math. Jussieu 2 (2003), no. 4, p. 583-634.

[63] - "The characteristic cycle and the singular support of a constructible sheaf", Invent. Math. 207 (2017), no. 2, p. 597-695, corrigendum in Invent. Math. 216 (2019), $\mathrm{n}^{\circ} 3$, p. $1005-1006$.

[64] - "Wild ramification and the cotangent bundle", J. Algebr. Geom. 26 (2017), no. 3, p. 399-473.

[65] S. Schieder, "Picard-Lefschetz oscillators for the Drinfeld-Lafforgue-Vinberg degeneration for SL2", Duke Math. J. 167 (2018), no. 5, p. 835-921.

[66] P. Scholze, "Perfectoid spaces", Publ. Math., Inst. Hautes Étud. Sci. 116 (2012), p. 245-313. 
[67] J.-P. Serre, Groupes algébriques et corps de classes, Actualités Scientifiques et Industrielles, Hermann, 1959, Publications de l'institut de mathématique de l'université de Nancago, VII, 202 pages.

[68] J.-P. Serre \& J. T. TAte, "Good reduction of abelian varieties", Ann. Math. 88 (1968), p. 492-517.

[69] J. Steenbrink, "Limits of Hodge structures", Invent. Math. 31 (1976), no. 3, p. 229257.

[70] S. Sun \& W. Zheng, "Parity and symmetry in intersection and ordinary cohomology", Algebra Number Theory 10 (2016), no. 2, p. 235-307.

[71] T. Suzuki, "Néron models of 1-motives and duality", Kodai Math. J. 42 (2019), no. 3, p. $431-475$.

[72] - "Grothendieck's pairing on Néron component groups: Galois descent from the semistable case", Kyoto J. Math. 60 (2020), no. 2, p. 593-716.

[73] J. T. Tate, "p-divisible groups", in Proc. Conf. Local Fields (Driebergen, 1966), Springer, 1967, p. 158-183.

[74] M. Temkin, "Stable modification of relative curves", J. Algebr. Geom. 19 (2010), no. 4, p. 603-677.

[75] N. Umezaki, "A uniform bound for the order of monodromy", Math. Res. Lett. 23 (2016), no. 3, p. 929-937.

[76] A. Werner, "On Grothendieck's pairing of component groups in the semistable reduction case", J. Reine Angew. Math. 486 (1997), p. 205-215. 